\title{
Shear stiffness of granular material at small strains: does it depend on grain size?
}

\author{
J. YANG ${ }^{*}$ and X. Q. GU*
}

\begin{abstract}
The shear stiffness of granular material at small strain levels is a subject of both theoretical and practical interest. This paper poses two fundamental questions that appear to be interrelated: (a) whether this stiffness property is dependent on particle size; and (b) whether the effect of testing method exists in terms of laboratory measurements using resonant column (RC) and bender element (BE) tests. For three uniformly graded types of glass beads of different mean sizes $(0 \cdot 195 \mathrm{~mm}$, $0.920 \mathrm{~mm}$ and $1.750 \mathrm{~mm}$ ), laboratory tests were conducted at a range of confining stresses and void ratios, using an apparatus that incorporates both $\mathrm{RC}$ and $\mathrm{BE}$ functions and thus allows reliable and insightful comparisons. It is shown that the small-strain stiffness, determined by either the RC or BE tests, does not vary appreciably with particle size, and it may be practically assumed to be size independent. The laboratory experiments also indicate that the $\mathrm{BE}$ measurements of small-strain stiffness are comparable to the corresponding RC measurements, with differences of less than $10 \%$. Furthermore, the BE measurements for fine glass beads are found to be consistently higher than the RC measurements, especially at large stress levels, whereas this feature becomes less evident for medium-coarse glass beads, and eventually diminishes for coarse glass beads. The study indicates that the characteristics of output signals in BE tests can be largely affected by the frequency of the input signal, the mean particle size of the material and the confining stress level, and that these factors are interrelated. Improper interpretation of wave signals may lead to shear stiffness measurements that are unreasonably low, either showing a substantial increase with particle size or showing the opposite. A micromechanics-based analysis assuming the Hertz-Mindlin contact law is presented to offer an understanding of the size effect from the grain scale.
\end{abstract}

KEYWORDS: dynamics; elasticity; laboratory equipment; laboratory tests; stiffness

\section{INTRODUCTION}

The shear stiffness of granular soils at strain levels less than $0.001 \%$ - usually denoted as $G_{0}$ or $G_{\max }$ - is a key parameter in major geotechnical applications involving deep excavations and tunnels, liquefaction evaluation or earthquake ground response analysis. Extensive research has been carried out to study this property, mainly through well-controlled laboratory experiments, particularly using resonant column (RC) tests as they offer high reliability and accuracy at this small strain level (Hardin \& Richart, 1963; Iwasaki \& Tatsuoka, 1977; Chung et al., 1984; Lo Presti et al., 1997). Of the factors identified as affecting shear stiffness, confining stress and void ratio are recognised to be the main ones. They are now commonly accounted for using an empirical formula taking the form

$$
G_{0}=A F(e)\left(\frac{\sigma^{\prime}}{p_{\mathrm{a}}}\right)^{n}
$$

where $G_{0}$ is in $\mathrm{MPa} ; \sigma^{\prime}$ is the effective confining stress in $\mathrm{kPa} ; p_{\mathrm{a}}$ is a reference stress, usually taken as the atmospheric pressure; $A$ and $n$ are two best-fit parameters; and $F(e)$ is a function of void ratio (e) with a typical form of (e.g. Hardin \& Richart, 1963; Iwasaki \& Tatsuoka, 1977)

Manuscript received 6 July 2011; revised manuscript accepted 17 May 2012. Published online ahead of print 16 October 2012.

Discussion on this paper closes on 1 July 2013, for further details see p. ii.

* Department of Civil Engineering, The University of Hong Kong, Hong Kong.

$$
F(e)=\frac{(2 \cdot 17-e)^{2}}{1+e}
$$

Recent notable work using the resonant column technique includes that by Wichtmann \& Triantafyllidis (2009), who conducted a structured programme of resonant column (RC) tests to examine the effect of particle-size distribution on the stiffness of sand. Their data showed that the value of $G_{0}$ decreased markedly as the coefficient of uniformity $\left(C_{\mathrm{u}}=D_{60} / D_{10}\right)$ of sand increased, where $D_{60}$ and $D_{10}$ represent the particle sizes that $60 \%$ and $10 \%$ of the sand mass are smaller than, respectively. This result is in agreement with that of Iwasaki \& Tatsuoka (1977), which was also derived from a number of $\mathrm{RC}$ tests on sand. The experimental data also suggested that the small-strain stiffness was independent of the mean particle size of sand $\left(D_{50}\right)$.

Bender element (BE) tests, which involve direct measurement of shear waves, have become a promising alternative in recent years for laboratory determination of $G_{0}$ values of soils (Dyvik \& Madshus, 1985; Viggiani \& Atkinson, 1995; Brignoli et al., 1996; Yamashita et al., 2009; Clayton, 2011). A significant advantage of this technique is that it can be incorporated in standard soil mechanics apparatuses such as triaxial and oedometer devices, and the approaches for data interpretation are relatively simple. Relying on this technique, Patel et al. (2008) measured the shear wave velocity $\left(V_{\mathrm{s}}\right)$ in assemblies of glass beads of different sizes. Their tests indicated that $V_{\mathrm{s}}$ increased as the mean particle size of glass beads decreased, as shown in Fig. 1(a). At the confining stress of $100 \mathrm{kPa}$, the $V_{\mathrm{s}}$ of glass beads with a mean diameter of $0.5 \mathrm{~mm}$ was determined to be $320 \mathrm{~m} / \mathrm{s}$, which was about $45 \%$ higher than that of glass beads with a mean diameter of $2.5 \mathrm{~mm}$. In terms of shear modulus, the $G_{0}$ 
value of the small-sized glass beads was about $110 \%$ higher than that of the large-sized glass beads.

The experimental work of Patel et al. (2008), showing an appreciable size dependence of small-strain stiffness, does not agree with that of Wichtmann \& Triantafyllidis (2009) and Iwasaki \& Tatsuoka (1977). It does, however, appear to be consistent with that of Bartake \& Singh (2007), who performed BE tests on three dry sands with similar gradation and found that the $G_{0}$ value increased as the mean particle size $\left(D_{50}\right)$ of the sand decreased. The work of Sharifipour et al. (2004), also using the BE technique to measure the shear wave velocity in glass beads, adds further uncertainty: for glass beads of three different nominal sizes $(1 \cdot 0,2 \cdot 0$ and $3.0 \mathrm{~mm}$ ), they obtained an opposite result, showing that the value of $V_{\mathrm{s}}$ increased with increasing particle size, as shown in Fig. 1(b). In terms of shear modulus, $3.0 \mathrm{~mm}$ glass beads at a confining stress of $100 \mathrm{kPa}$ were determined to have a $G_{0}$ value of about $111.2 \mathrm{MPa}$, being $66 \%$ larger than that of $1.0 \mathrm{~mm}$ glass beads.

Evidently, the issue of whether the small-strain shear stiffness is size dependent remains inconclusive and open to discussion. Also, the experimental data from BE tests in the literature seem always to indicate that particle size has an effect on small-strain shear stiffness (although opposite trends were observed in the $G_{0}$ variation with grain size), whereas the RC test data seem always to suggest that there is not any size effect. In this respect, an additional question arises: does the testing method have any effect on smallstrain stiffness? In RC tests, a soil specimen is subjected to torsional excitation typically at frequencies of several tens of $\mathrm{Hz}$, and the shear stiffness $\left(G_{0}\right)$ is estimated from the measured resonant frequency. In BE tests, however, the shear wave velocity in a soil specimen $\left(V_{\mathrm{s}}\right)$ is measured directly

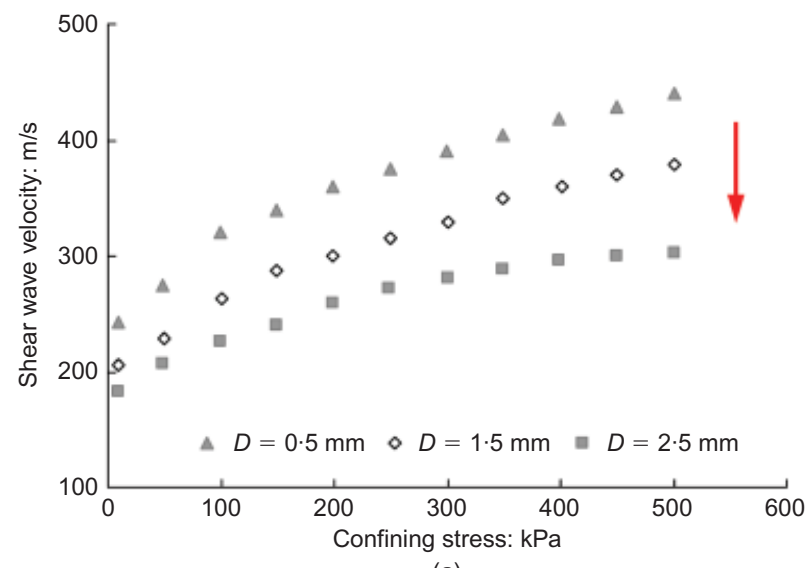

(a)

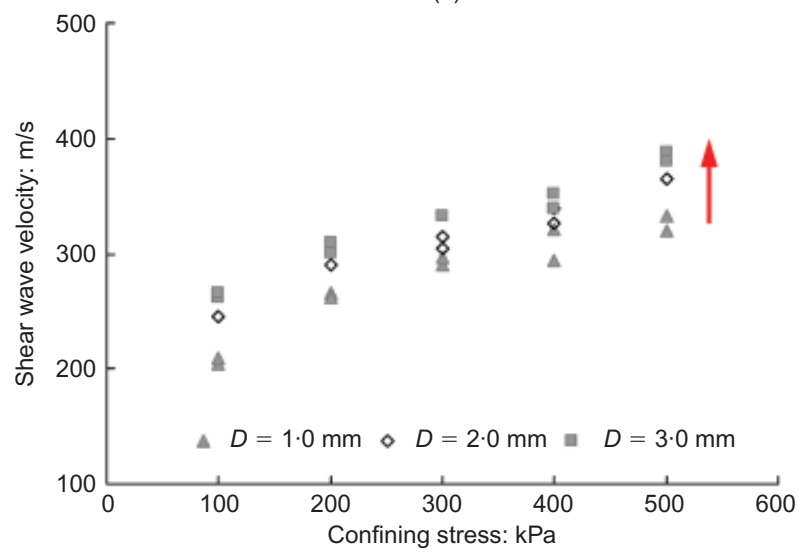

(b)

Fig. 1. Test data on shear wave velocity in glass beads of different sizes: (a) Patel et al. (2008); (b) Sharifipour et al. (2004) through a pair of piezoelectric transducers at frequencies from several to a few tens of $\mathrm{kHz}$, and is then converted to the shear modulus by $G_{0}=\rho\left(V_{\mathrm{s}}\right)^{2}$, where $\rho$ is the mass density of the soil. The two methods indeed involve different principles and interpretations.

With the aim of addressing these two fundamentally important questions, a specifically designed experimental programme has been carried out using an apparatus that incorporates both $\mathrm{RC}$ and $\mathrm{BE}$ features. The apparatus allows the $\mathrm{BE}$ and $\mathrm{RC}$ tests to be performed on an identical specimen, thus affording more reliable and convincing comparisons; this is both necessary and desirable, given the observed discrepancies between the $\mathrm{RC}$ and $\mathrm{BE}$ measurements. Three types of glass beads with different mean particle sizes $\left(D_{50}\right)$ but the same uniformity $\left(C_{\mathrm{u}}\right)$ were prepared and used as analogue granular soils in the experiment. Their simple and spherical geometry, together with the same uniformity, allowed the influences of particle shape (Cho et al., 2006) and particle size distribution (Wichtmann \& Triantafyllidis, 2009) to be isolated, so that any observed difference is attributable to the difference in particle size. This paper presents the main results of this experimental work. Moreover, efforts are made here to explore the possible causes of the contradictory results in the literature through a comprehensive analysis of the experimental data. A grain-scale model is also presented to provide an understanding from the micromechanical point of view.

\section{TEST EQUIPMENT AND MATERIAL \\ Equipment set-up}

The overall set-up of the apparatus is schematically shown in Fig. 2, with close-up views of the key components given in Fig. 3. The apparatus has both $\mathrm{RC}$ and $\mathrm{BE}$ features and a robust signal conditioning and data acquisition system, along with an environmental chamber. It can accommodate a soil specimen $50 \mathrm{~mm}$ in diameter and $100 \mathrm{~mm}$ high, with an airfilled cell pressure up to $1 \mathrm{MPa}$. The resonant column is of bottom-fixed and top-free configuration (the Stokoe type). Compared with the free-free configuration, the fixed-free configuration has the advantages of relatively high available torque and convenient access to the specimen for control of effective stress. A careful calibration of the equipment was carried out using three aluminium bars of different dimensions to establish a calibration curve for the frequencydependent mass polar moment of inertia of the drive head. Attention should be paid to specimen fixity when testing very stiff materials such as highly cemented sand or weak rock; more details of this issue are beyond the scope of this paper but can be found in, for example, Clayton et al. (2009).

To allow for the pulse test in the same system, a pair of bender elements has been instrumented, as shown in Fig. 3. Unlike the conventional design, this single pair of bender elements is able to generate not only shear waves (i.e. S-waves) but also compression waves (i.e. P-waves); this has been achieved by modifying the wiring configuration of the bender elements (Lings \& Greening, 2001). Measuring the compression wave can facilitate the interpretation of shear wave signals in situations where the so-called near-field effect is complicated, and it may also serve as a promising alternative for checking the degree of saturation of soil specimens (Yang, 2002). A careful calibration of the pulse testing system was carried out by putting the tips of the two bender elements in direct contact to determine the system delay, including the response time of bender elements and the travel time in the cables. The phase relationship (i.e. initial polarisations) between the input and output signals was also checked in the calibration. 


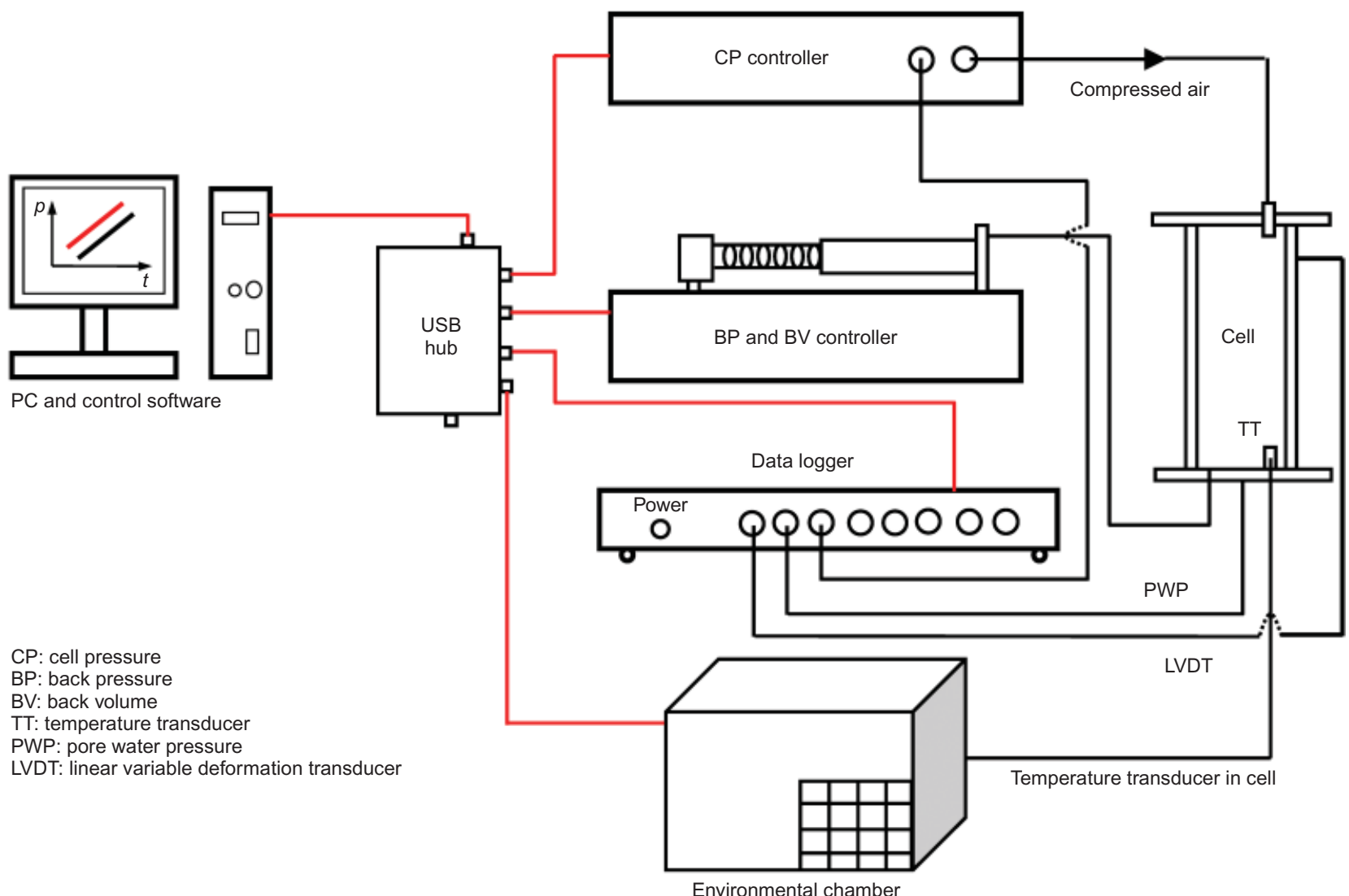

Fig. 2. Set-up of dynamic testing system at The University of Hong Kong
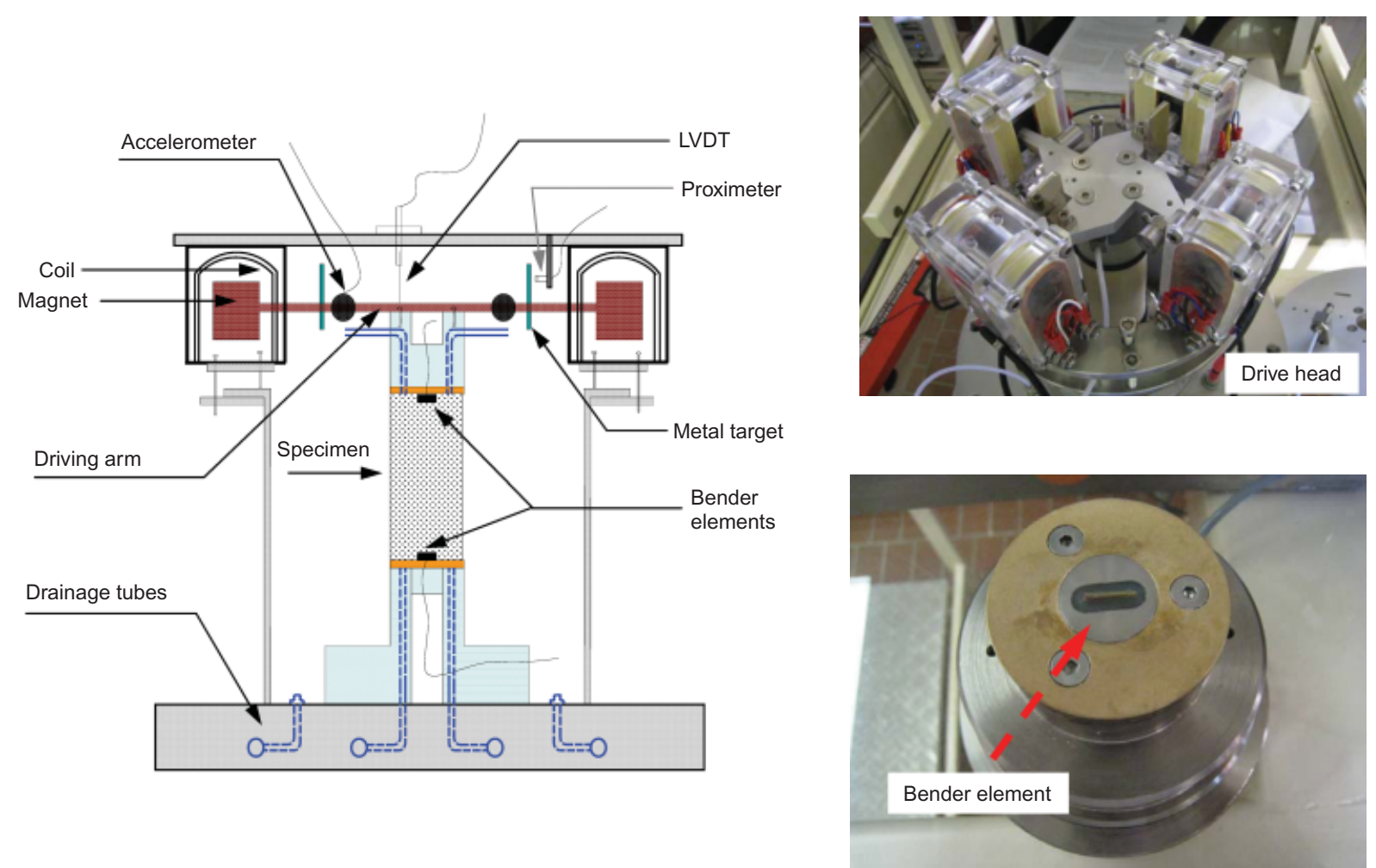

Fig. 3. Close-up views of apparatus (not to scale)

\section{Test material and sample preparation}

Three types of uniformly graded glass beads were prepared for the experiment. Fig. 4 shows their size distribution curves, along with a scanning electron microscopy (SEM) photograph showing the shape of the particles. Glass beads of type A, denoted GB-A, were the coarsest, with a mean particle size $\left(D_{50}\right)$ of $1.750 \mathrm{~mm}$; glass beads of type D (GBD) were the finest, with a $D_{50}$ of $0.195 \mathrm{~mm}$; and glass beads of type B (GB-B) with a $D_{50}$ of $0.920 \mathrm{~mm}$ were in between (Table 1). The range of particle size was reasonably wide to cover the sizes of fine to coarse sand, and all three types of glass beads shared a similar gradation $\left(C_{\mathrm{u}}=1.22-1.25\right)$ 


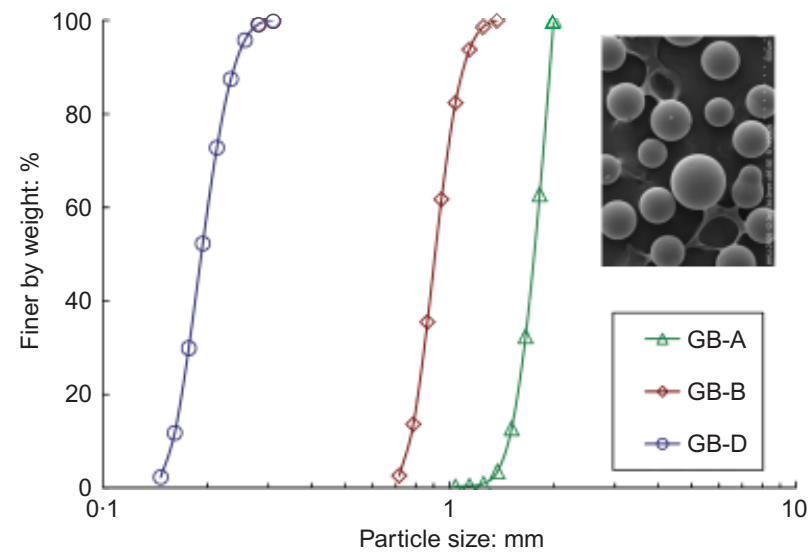

Fig. 4. Particle-size distribution curves of glass beads used in experiments

Table 1. Physical properties of glass beads tested

\begin{tabular}{l|c|c|c|c|c}
\hline Glass bead & $G_{\mathrm{s}}$ & $D_{10}: \mathrm{mm}$ & $D_{50}: \mathrm{mm}$ & $D_{60}: \mathrm{mm}$ & $C_{\mathrm{u}}$ \\
\hline GB-A & $2 \cdot 462$ & $1 \cdot 48$ & $1 \cdot 750$ & $1 \cdot 81$ & $1 \cdot 22$ \\
GB-B & $2 \cdot 462$ & $0 \cdot 77$ & 0.920 & $0 \cdot 94$ & $1 \cdot 22$ \\
GB-D & $2 \cdot 462$ & $0 \cdot 16$ & $0 \cdot 195$ & $0 \cdot 20$ & $1 \cdot 25$ \\
\hline
\end{tabular}

such that the influence of uniformity was isolated. The uniformity of the glass beads is comparable to that of Toyoura sand (1.392), tested by Yang \& Gu (2010) using the same apparatus.

Industrially made glass beads may not be perfectly spherical. This defect in shape regularity was observed in all three types of glass beads. From a statistical point of view, however, it can be assumed that these glass beads have a similar roundness and surface roughness, and the potential effect of shape defect or the effect of difference in surface roughness on test results is minor.

Prior to the stiffness measurements, the glass beads were oven-dried and then cooled in sealed containers to remove the potential influence of moisture on the particle surface. All specimens in the tests were prepared using the dry tamping method in five layers. The method involved pouring the material using a funnel without falling height and then performing compaction using a tamper. Note that in the preparation of specimens at the loose state, no significant tamping was used. To stand the specimens and support the weight of the drive arm of the resonant column, a suction of $25 \mathrm{kPa}$ was applied to the specimens. The cell pressure was then increased and the suction was decreased simultaneously to keep a constant isotropic effective confining stress of $25 \mathrm{kPa}$, which was taken as the initial stress level of the specimens.

\section{Test series}

For each type of glass beads a set of specimens were prepared in different initial packing states: loose $(e=0.595$ $0.623)$, medium-dense $(e=0.575-0.587)$ and dense $(e=$ $0.558-0.567)$ states. For each packing density, the BE and $\mathrm{RC}$ tests were conducted at the confining stresses of 50, 100, 200 and $400 \mathrm{kPa}$ in sequence. In bringing the specimens to a specific stress level, each specimen was first consolidated for $15 \mathrm{~min}$ at this stress level, and the corresponding deformation was measured by the internal high-resolution linear variable differential transducer (LVDT) (the reading of the LVDT usually became stable within the time allowed); then the $\mathrm{BE}$ test was performed under a range of excitation frequencies. Following the $\mathrm{BE}$ test, the $\mathrm{RC}$ test was then performed on the same specimen for the purpose of comparison of the stiffness measurements. Table 2 summarises the test series.

\section{BENDER ELEMENT MEASUREMENTS}

Despite the increasing popularity of BE tests, considerable uncertainty remains in signal interpretation, and thus in the estimated shear stiffness. Clayton (2011) showed a good example of large scatter in shear wave velocities estimated from a single $\mathrm{BE}$ test on a specimen of natural clay, commenting that previous estimates of the accuracy of $\mathrm{BE}$ measurements have been optimistic. This opinion is supported by the largely scattered results of the international parallel BE tests on uniform Toyoura sand (Yamashita et al., 2009). These observations, together with the contradictory results in the literature for stiffness variation with particle size, underscore the need for careful examination of $\mathrm{BE}$ tests, particularly for granular material, in which wave propagation is complex owing to its particulate nature. Here, efforts are made to clarify several issues that have not yet been extensively studied but are closely related to the reliability of BE measurements: (a) the characteristics of received signals in both the time and frequency domains over a wide range of excitation frequencies and wavelengths; (b) how changes of particle size alter the characteristics of received signals; and (c) the performance of different interpretation methods under a variety of combinations of test conditions (i.e. grain size, excitation frequency and confining stress).

\section{Effect of frequency on waveforms}

Figure 5 shows the waveforms generated in a specimen of coarse glass beads (GB-A, $D_{50}=1.750 \mathrm{~mm}$ ) by one cycle of sinusoidal signal at different excitation frequencies. The specimen was at an isotropic confining stress of $100 \mathrm{kPa}$ and a void ratio of 0.584 . The excitation frequencies covered a wide range, varying from $1 \mathrm{kHz}$ to as high as $40 \mathrm{kHz}$, thus allowing a systematic examination of the frequency effect. The dashed line indicates the travel time of the shear wave in the specimen, deduced from the shear wave velocity determined by the RC test. By comparison, the upward triangle indicates the first arrival of the shear wave based on the waveform at the frequency of $10 \mathrm{kHz}$. Evidently, as the excitation frequency increases, the received signal tends to contain more high-frequency components. At very high excitation frequencies $(20 \mathrm{kHz}$ and $40 \mathrm{kHz})$, the waveforms become quite similar in shape. In addition, the initial signal component with negative polarity preceding the arrival of the major components - marked by a downward triangle and representing the near-field effect - appears to be strongest at the excitation frequency of $1 \mathrm{kHz}$, and tends to fade as the excitation frequency increases.

Similar observations have been obtained on a mediumcoarse specimen (GB-B, $\left.D_{50}=0.920 \mathrm{~mm}\right)$, which was also at an isotropic confinement of $100 \mathrm{kPa}$ and a void ratio of $0 \cdot 585$, and was also excited by a sinusoidal input of varying frequencies (Fig. 6). Compared with the waveforms shown in Fig. 5, the received signal at each corresponding frequency contains components of higher frequencies, implying that a change in particle size can affect the frequency content of the received signal. This feature becomes more evident in Fig. 7, where the waveforms in a fine specimen (GB-D, $D_{50}=0.195 \mathrm{~mm}$ ) under various excitation frequencies are shown. Also, it is to be noted that when the grain size reduces from $1.750 \mathrm{~mm}$ to $0.195 \mathrm{~mm}$, the initial component with negative polarity tends to fade as well. 
Table 2. Summary of test series

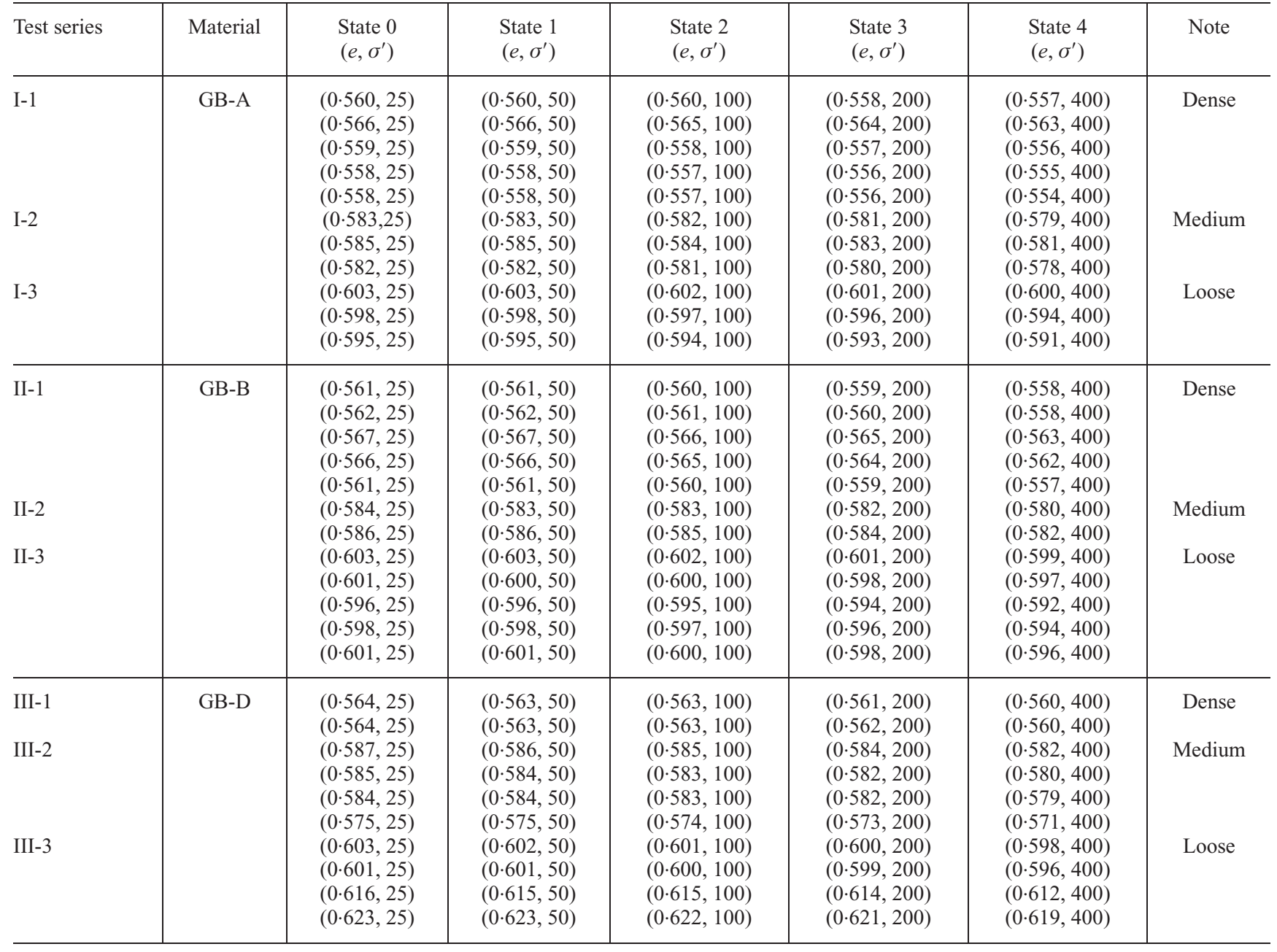

Note: $e=$ void ratio; $\sigma^{\prime}=$ effective confining stress (in $\mathrm{kPa}$ ); state $0=$ initial state.

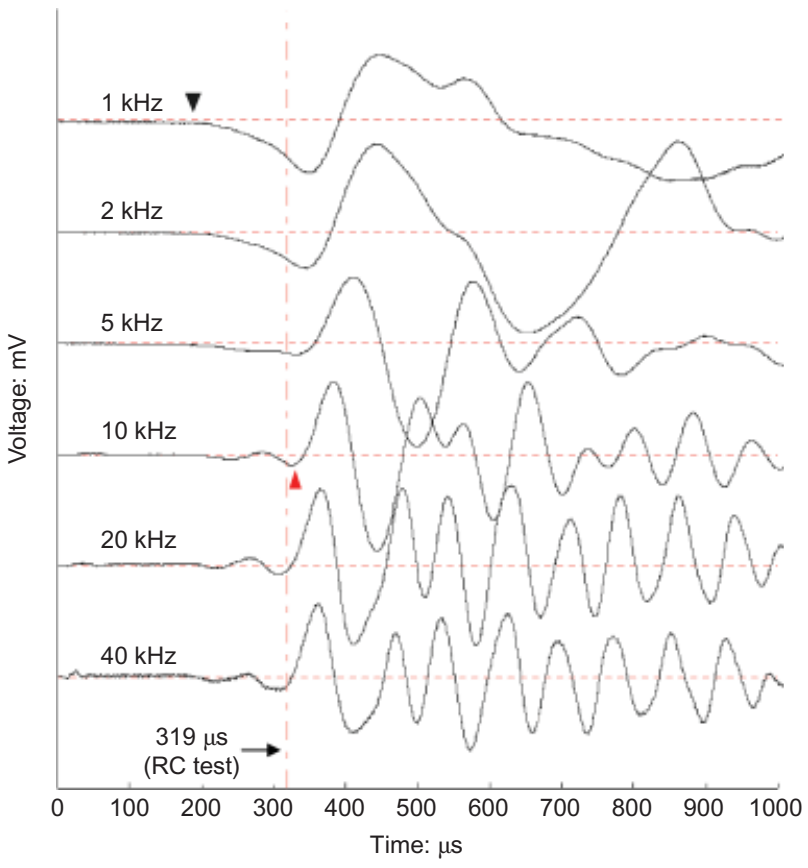

Fig. 5. Shear wave signals in glass beads GB-A $\left(D_{50}=1 \cdot 750 \mathrm{~mm}\right)$ at various excitation frequencies (sinusoidal input, $\sigma^{\prime}=100 \mathrm{kPa}$; $e=0 \cdot 584)$

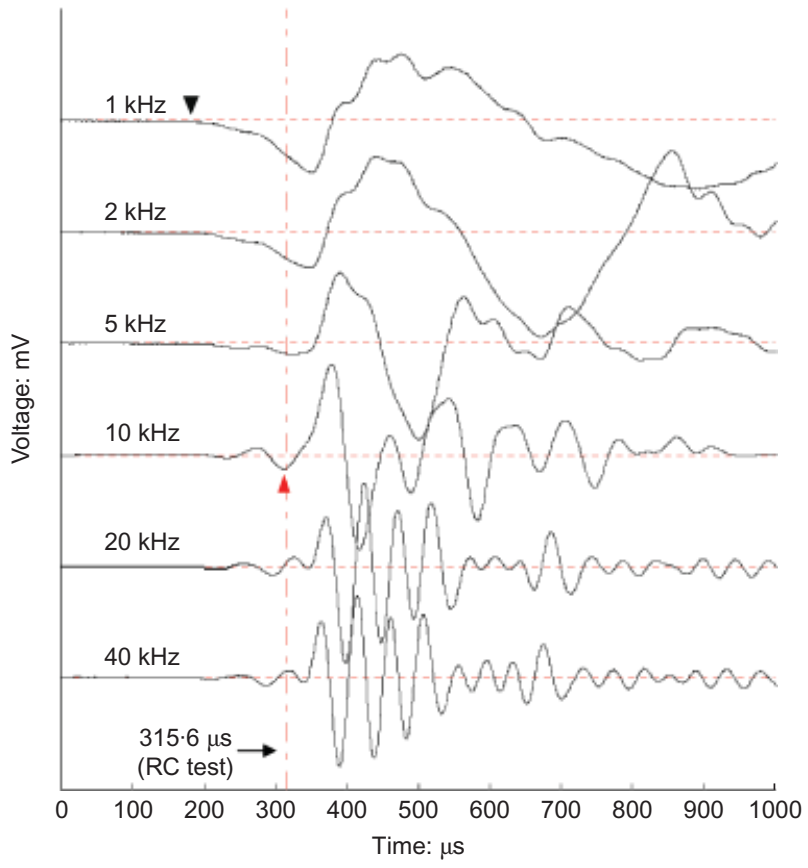

Fig. 6. Shear wave signals in glass beads GB-B $\left(D_{50}=0.920 \mathrm{~mm}\right)$ at various excitation frequencies (sinusoidal input, $\sigma^{\prime}=100 \mathrm{kPa}$; $e=0 \cdot 585$ ) 


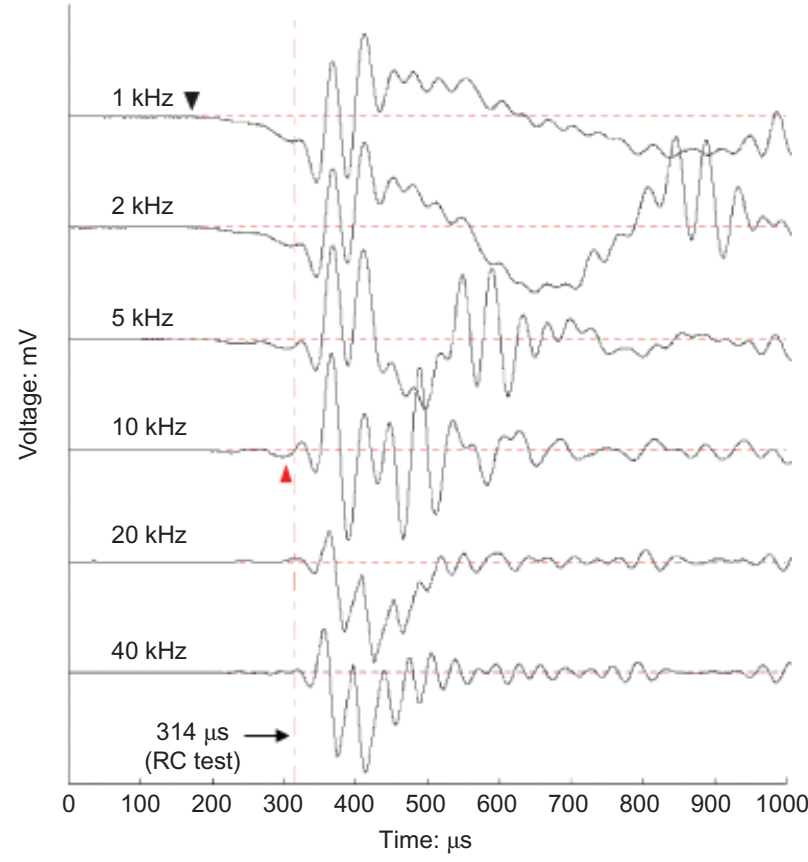

Fig. 7. Shear wave signals in glass beads GB-D $\left(D_{50}=0 \cdot 195 \mathrm{~mm}\right)$ at various excitation frequencies (sinusoidal input, $\sigma^{\prime}=100 \mathrm{kPa}$; $e=0 \cdot 583$ )

\section{Effect of particle size on waveforms}

To allow a better identification of the importance of particle size in modifying waveforms, Fig. 8 compares the waveforms generated in the three specimens under otherwise similar conditions. The waveforms at the excitation frequency of $5 \mathrm{kHz}$ are compared in Fig. 8(a), and another three waveforms at the excitation frequency of $10 \mathrm{kHz}$ are compared in Fig. 8(b). In either case of excitation frequency, a decrease in grain size can introduce high-frequency components to the output signal. The waveforms in the finest specimen GB-D, at either $5 \mathrm{kHz}$ or $10 \mathrm{kHz}$, exhibit a first peak with an amplitude that is much less than the subsequent largest peak. It is the excursion of this small peak that represents the true arrival of the shear wave (marked by an upward triangle on the waveform for specimen GB-D in Fig. 8(b)). For purposes of comparison, the travel times of the shear waves in the three specimens were deduced from the RC measurements, and are marked by three downward dashed arrows on the corresponding waveforms. It becomes clear that, if the excursion of the largest peak is selected as the arrival of the shear wave (marked by the indicator ' $x$ '), a stiffness value substantially lower than the RC measurement will be yielded.

The existence of a small-amplitude peak preceding the largest peak in the shear wave signal was also reported by Brignoli et al. (1996) in pulse tests on uniform Ticino sand $\left(D_{50}=0.710 \mathrm{~mm}\right)$; it was also observed in testing Toyoura sand $\left(D_{50}=0.216 \mathrm{~mm}\right)$ using the same apparatus (Yang \& $\mathrm{Gu}, 2010)$, as shown in Fig. 9 for comparison. This feature is not evident in the waveforms generated in the coarsest specimen (GB-A; Fig. 5), but for the medium-coarse specimen (GB-B) the feature tends to appear when the excitation frequency is sufficiently high (Fig. 6). It is also found that, for a given excitation frequency, increasing the confining stress to large levels tends to result in the occurrence of the small peak, as shown in Fig. 10 for glass beads GB-B.

The waveforms in Figs 5-8 and the discussion above show the important finding that the effect of particle size is coupled with the effect of frequency in altering the characteristics of waveforms. This is understandable, given the parti-

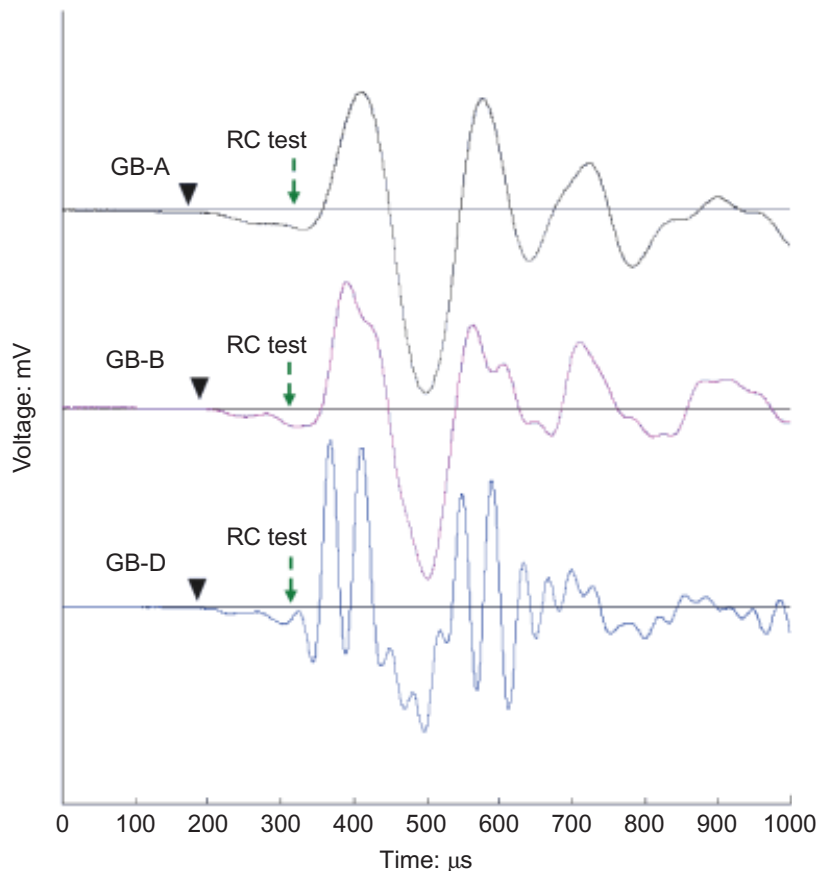

(a)

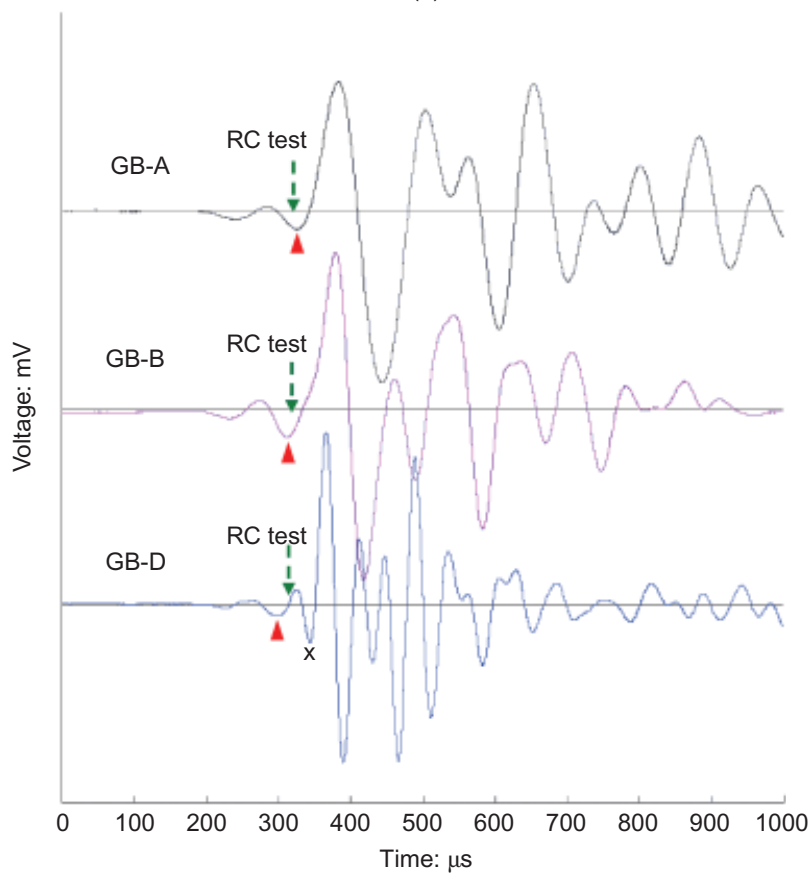

(b)

Fig. 8. Shear wave signals in glass beads of different sizes (sinusoidal input, $\sigma^{\prime}=100 \mathrm{kPa} ; e \approx 0.584$ ): (a) excitation frequency $=5 \mathrm{kHz}$; (b) excitation frequency $=10 \mathrm{kHz}$

culate nature of the material. To explore their relation, the fast Fourier transform was conducted for the output signals in Figs 5-7, and the predominant frequency was identified for each signal. Fig. 11 shows this predominant frequency, denoted as $f_{\text {out }}$, as a function of the excitation frequency $\left(f_{\text {in }}\right)$ for the three specimens, together with the trend lines. There are several features in Fig. 11 that are worth noting. First, for either coarse or fine specimens, the predominant frequency tends to increase with excitation frequency in an approximately linear manner in a low-frequency range, and then tends to approach a limiting value at high excitation frequencies. In this respect, a threshold frequency marking the transition can be identified. Second, this threshold frequency appears to depend on particle size, in that it takes larger 


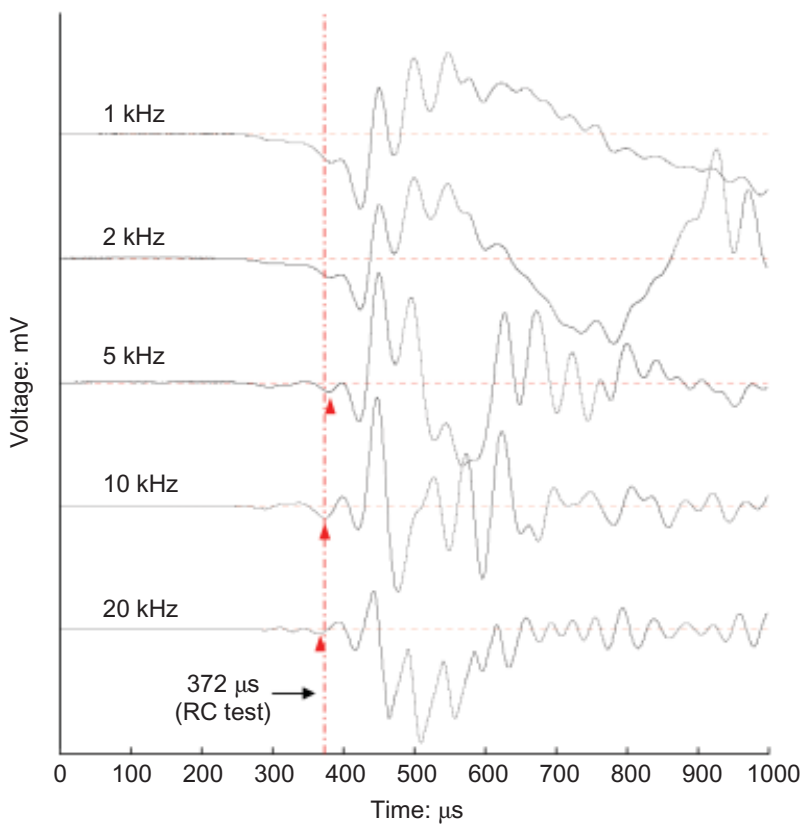

Fig. 9. Shear wave signals in dry Toyoura sand at various excitation frequencies $\left(\sigma^{\prime}=100 \mathrm{kPa} ; e=0 \cdot 798\right)$

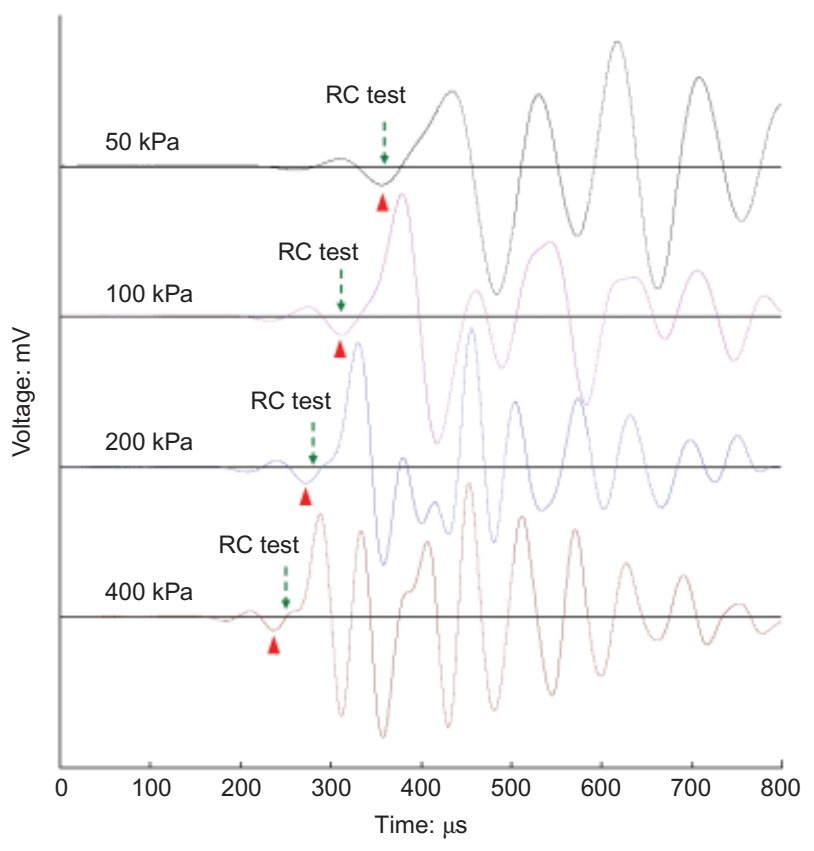

Fig. 10. Shear wave signals in glass beads GB-B at various confining stresses (sinusoidal input, $e \approx 0 \mathbf{0} 585$ )

values for fine specimens or smaller values for coarse specimens. For example, for specimen GB-A the threshold frequency is around $16 \mathrm{kHz}$, whereas it is above $20 \mathrm{kHz}$ for specimen GB-D. Third, the predominant frequency does not appear to be sensitive to particle size in the range of low excitation frequency (say, below $5 \mathrm{kHz}$ ); however, as the excitation frequency is further increased, it tends to become larger for fine specimens than for coarse specimens. Moreover, the limiting value of the predominant frequency at high excitation frequencies seems to increase with decreasing particle size. The interesting features described above are based on the experiments conducted on uniform glass beads; further validation using laboratory tests on different granular materials would be of benefit.

The experimental data described above offer evidence that

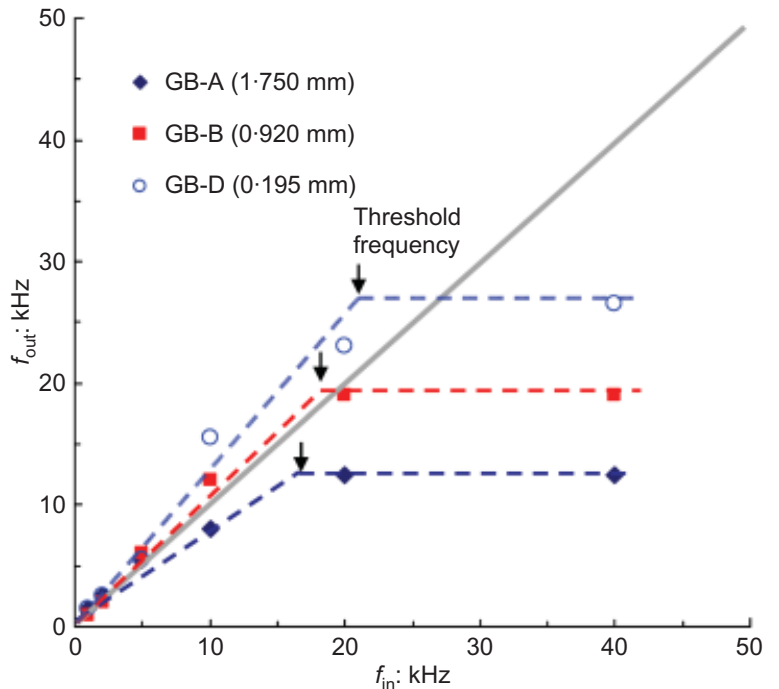

Fig. 11. Output signal frequency against input signal frequency

the shear wave signal generated in a granular sample, even with a simple sinusoidal input, can be very complicated. Its characteristics depend on both excitation frequency and particle size, and these factors are interrelated. With regard to shear stiffness measurement, the important implication is that diverse or even contradictory results may be yielded on the shear wave velocity $\left(V_{\mathrm{s}}\right)$ or the small-strain stiffness $\left(G_{0}\right)$ if these factors are not properly taken into account in data interpretation; this will be elaborated in more detail in the following section.

\section{Evaluation of various interpretation methods}

The largest uncertainty and difficulty with BE tests lie in determination of the travel time of the shear wave (Jovicic et al., 1996; Lee \& Santamarina, 2005; Yamashita et al., 2009). The commonly used approaches, as documented in Yamashita et al. (2009), are generally simplistic, and do not well recognise that the boundary conditions imposed on the specimen can lead to considerable divergence from the simplified solutions. One of the common approaches is known as the start-to-start method and another is the peakto-peak method, both working in the time domain. The idea of the start-to-start method is to find the first arrival of the shear wave by visual inspection of the received signal. A number of characteristic points have been proposed in the literature as indicators for the first arrival of the shear wave. A systematic examination of differences in the estimated shear stiffness using these points has been conducted for both fine and coarse specimens, with particular attention paid to the size effect. To facilitate discussion, a typical waveform is shown in Fig. 12, with all possible characteristic points marked as the first arrival of the shear wave, including the first inflection S1, the troughs S2 and S4, and the zero intercepts S3 and S5.

The peak-to-peak method applies typically to a sinusoidal input in which the travel time is defined as the difference between the peak of the input signal and that of the output signal. As the received signal usually contains multiple peaks rather than a single peak, a common practice is to take the largest peak to estimate the travel time (P2 in Fig. 12). However, as will be further explored later, in many cases this largest peak does not offer a reasonable reference for determining the travel time of the shear wave; as an alternative, a smaller peak preceding the maximum one (P1 in Fig. 12) is also used for comparison purposes.

To overcome the subjectivity and uncertainty involved in 
172

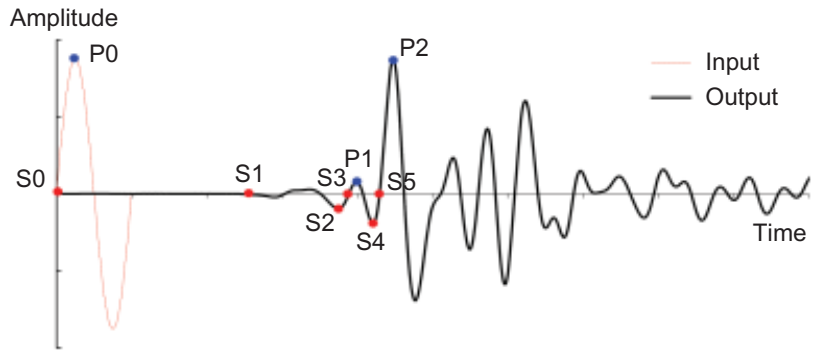

Fig. 12. Characteristic points for travel time interpretation

the time-domain methods, Viggiani \& Atkinson (1995) proposed the cross-correlation method, which works on the correlation of the input and received signals. The underlying assumption is that the two signals are of the same shape and frequency; however, this is not the case in BE tests and thus leads to significant errors, as shown later. In this method the travel time of the shear wave is defined at the position corresponding to the largest peak of the correlation, as shown schematically in Fig. 13 (CC-2). Because the time histories of the correlation contain multiple peaks, an alternative is to select the first, small-amplitude peak (CC-1) to determine the travel time.

Using the various methods described above, values of $G_{0}$ were determined for specimens GB-A, GB-B and GB-D under a confining stress of $100 \mathrm{kPa}$ and a void ratio of about 0.584 , for a wide range of excitation frequencies. The results are plotted in Fig. 14 as a function of excitation frequency. For purposes of comparison, the $G_{0}$ value determined by the resonant column test for each specimen is given as a benchmark, and two boundary lines marking $110 \%$ and $90 \%$ of this benchmark value are also given (i.e. $\pm 10 \%$ variations).

The three plots in Fig. 14 suggest the following.

(a) The $G_{0}$ values determined using any one of the methods appear to be frequency dependent: that is, the estimated $G_{0}$ value generally varies with excitation frequency.

(b) For a given excitation frequency, different interpretation methods yield different $G_{0}$ values, and the range of these values varies when particle size varies.

(c) The start-to-start method using point $\mathrm{S} 1$ always gives $G_{0}$ values that are unreasonably high in comparison with the $\mathrm{RC}$ measurements (the data points are all beyond the scale of the graphs), suggesting that point S1 does not correspond to the first arrival of the shear wave; it actually corresponds to the compression wave, as confirmed by the velocity measurement of this type of wave.

(d) The cross-correlation method, regardless of whether the largest peak (CC-2) or the first peak (CC-1) is used, usually yields $G_{0}$ values that are unreasonably low, particularly for coarse specimens GB-A and GB-B at high frequencies.

To better identify the effect of frequency, a ratio of travel distance $\left(L_{\mathrm{tt}}\right)$ and wavelength $(\lambda)$ is introduced as follows.

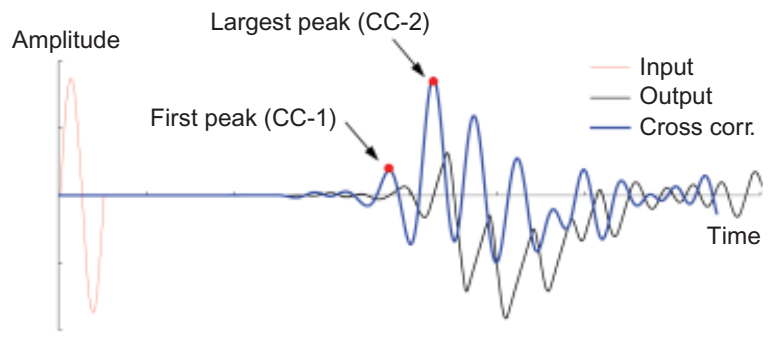

Fig. 13. Selection of peaks in cross-correlation method

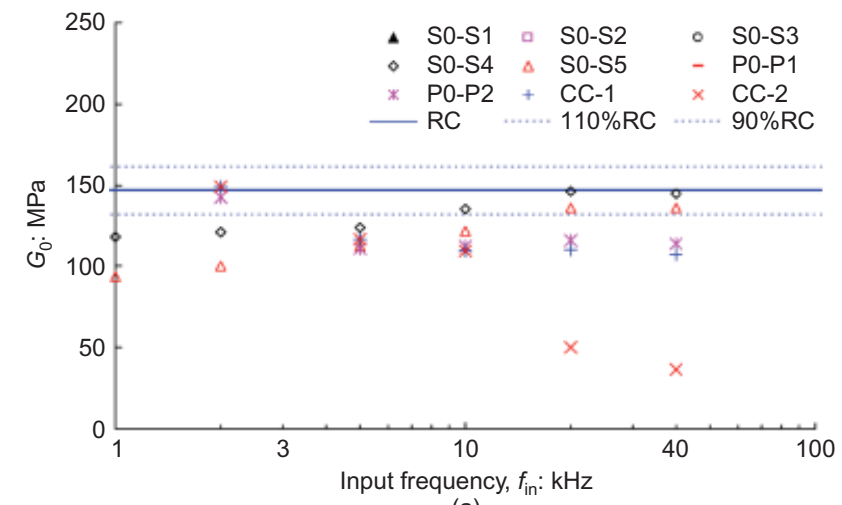

(a)
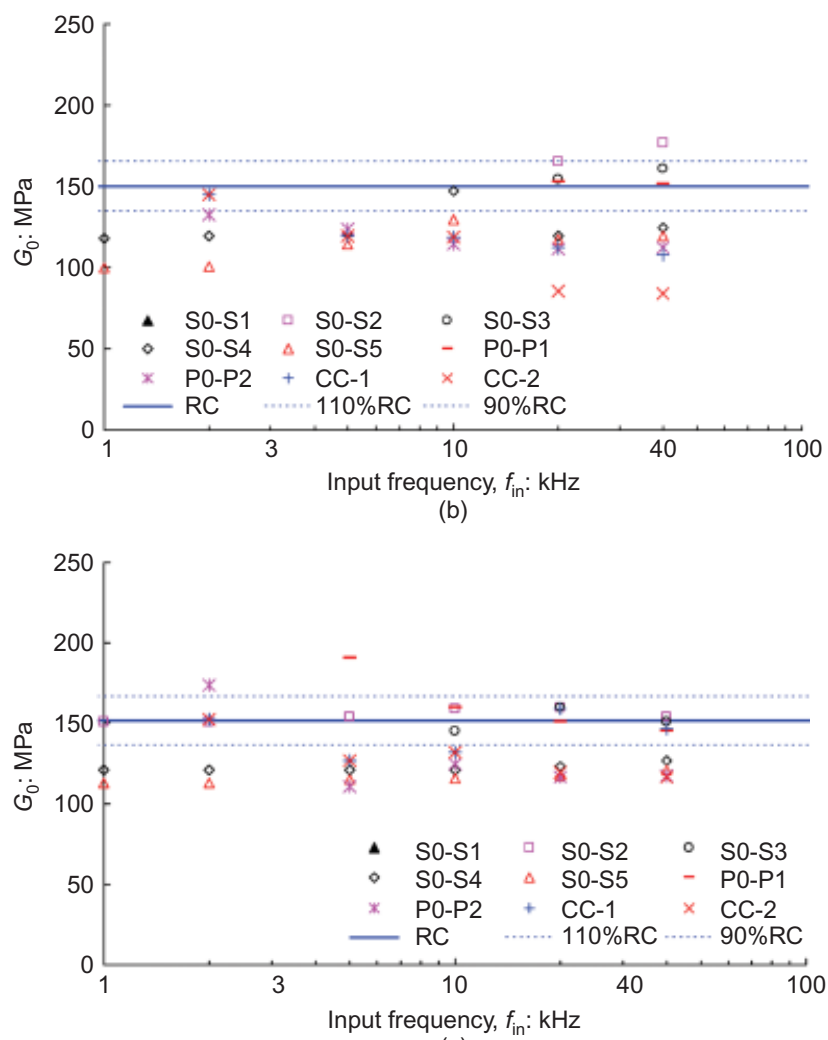

(c)

Fig. 14. Shear stiffness values estimated using various interpretation methods as a function of excitation frequency $\left(\sigma^{\prime}=100 \mathrm{kPa}\right)$ : (a) GB-A, $e=0.584$; (b) GB-B, $e=0.585$; (c) GB-D, $e=0.583$

$$
R_{\mathrm{d}}^{\mathrm{in}}=\frac{L_{\mathrm{tt}}}{\lambda}=\frac{L_{\mathrm{tt}}}{V_{\mathrm{s}}} f_{\text {in }}
$$

where the wavelength is calculated using the frequency of the sinusoidal input $\left(f_{\text {in }}\right)$, and the shear wave velocity is taken as that provided by the RC test. For each specimen the $G_{0}$ values from BE measurements were normalised by the RC measurement, and are shown as a function of the $R_{\mathrm{d}}^{\text {in }}$ ratio in Fig. 15. There are several points that are worth noting.

(a) The degree of scatter in $G_{0}$ values due to different interpretation methods is dependent on the $R_{\mathrm{d}}^{\text {in }}$ ratio, and it appears to be smallest for $R_{\mathrm{d}}^{\text {in }}$ values ranging from 2 to 4. This finding is consistent with the theoretical analysis of Sanchez-Salinero et al. (1986) that at least two wavelengths should be maintained between the transmitter and receiver; but it should be noted that much higher $R_{\mathrm{d}}^{\text {in }}$ values do not seem to help reduce uncertainty or increase accuracy.

(b) In this optimal range of $R_{\mathrm{d}}^{\text {in }}$ values, the start-to-start 


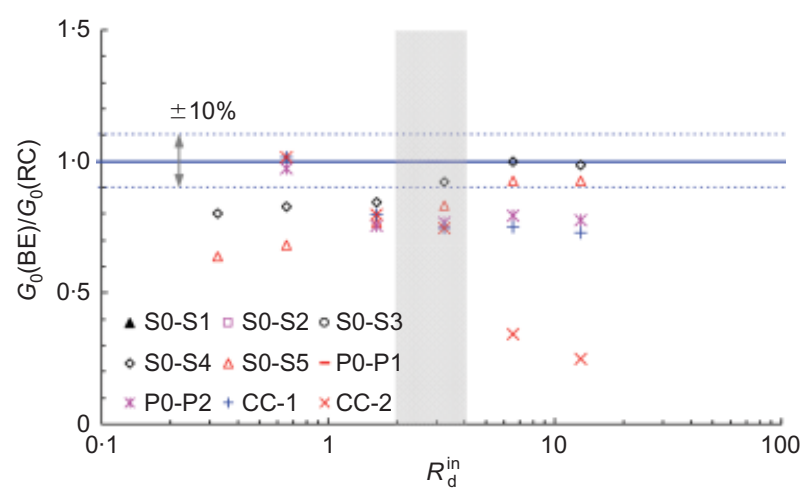

(a)

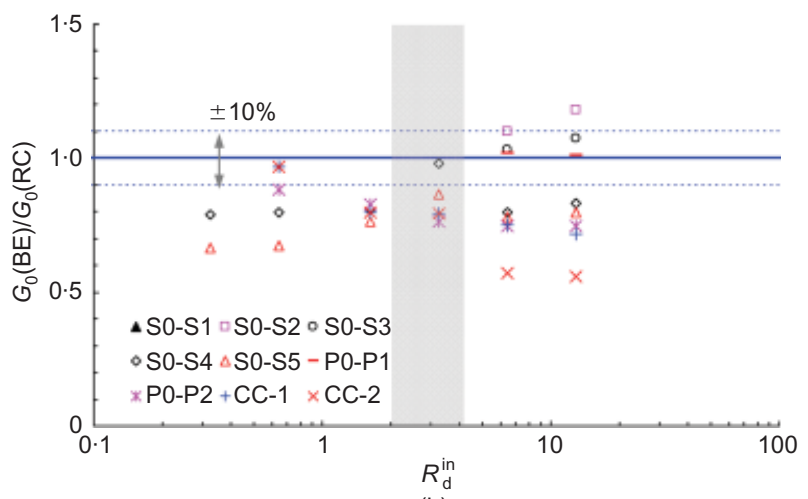

(b)

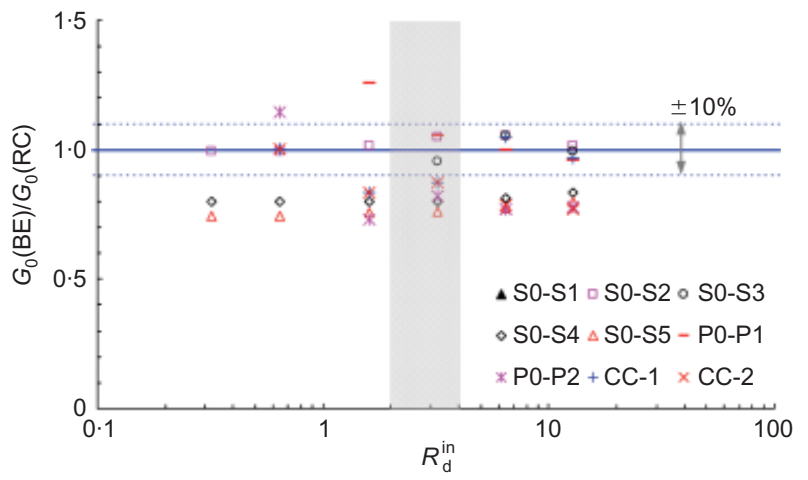

(c)

Fig. 15. Shear stiffness values estimated using various interpretation methods as a function of ratio between travel distance and wavelength $\left(\sigma^{\prime}=100 \mathrm{kPa}\right)$ : (a) GB-A, $e=0.584$; (b) GB-B, $e=$ 0.585; (c) GB-D, $e=0.583$

method using point S2 seems to work well for the fine specimen GB-D, whereas the start-to-start method using point S4 seems to work well for the coarse specimens GB-A and GB-B, for which point S2 appears to merge with point S4 (see Fig. 8(b)).

(c) The performance of the cross-correlation method using either CC-1 or CC-2 is improved in this optimal $R_{\mathrm{d}}^{\text {in }}$ range, but the method still provides $G_{0}$ values that are more than $20 \%$ lower than the corresponding RC measurements.

Bearing in mind the coupled effects of excitation frequency and particle size, a new index is introduced as the ratio between wavelength $(\lambda)$ and mean particle size $\left(D_{50}\right)$, and variations of the normalised $G_{0}$ values with this index are examined in Fig. 16. To facilitate comparison, the data shown in Fig. 16(a) were all generated using the start-tostart method, the data in Fig. 16(b) were generated using the peak-to-peak method, and Fig. 16(c) was produced using the cross-correlation method. Note that the wavelength was calculated using the frequency of the input sinusoidal signal and the RC reference value for shear wave velocity.

The three plots in Fig. 16 offer several important findings.

(a) The start-to-start method using either point S5 or S4 tends to give $G_{0}$ values that decrease as the $\lambda / D_{50}$ ratio increases: that is, for a given excitation frequency, the shear stiffness will decrease with decreasing particle size.

(b) The peak-to-peak method using $\mathrm{P} 0-\mathrm{P} 2$ (i.e. the largest peak) tends to give $G_{0}$ values that increase as the $\lambda / D_{50}$ ratio increases; this means that, for a given excitation frequency, the shear stiffness increases with decreasing particle size.

(c) The cross-correlation method also produces $G_{0}$ values that increase with increasing $\lambda / D_{50}$ ratio, either due to a decrease in particle size or to a reduction of excitation frequency. Also, the start-to-start method using point S2 is able to consistently provide reasonable $G_{0}$ values over a wide range of $\lambda / D_{50}$ ratios $(20-1000)$. These $G_{0}$ values are slightly greater than the $\mathrm{RC}$ benchmark values, and

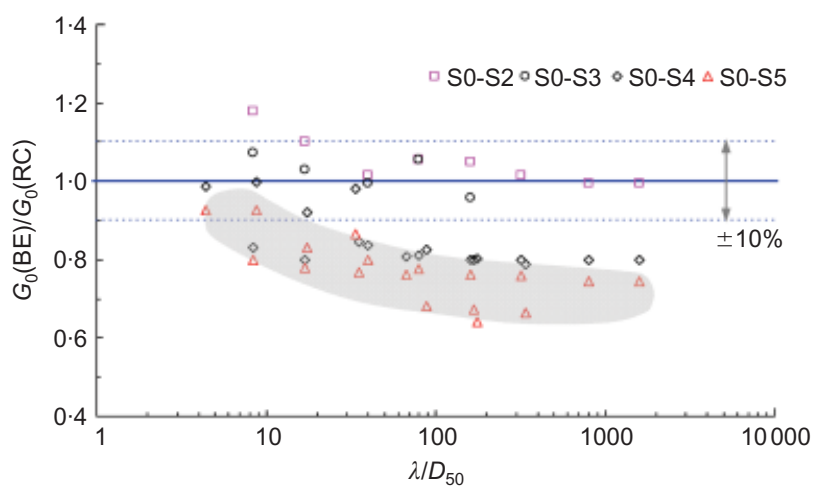

(a)

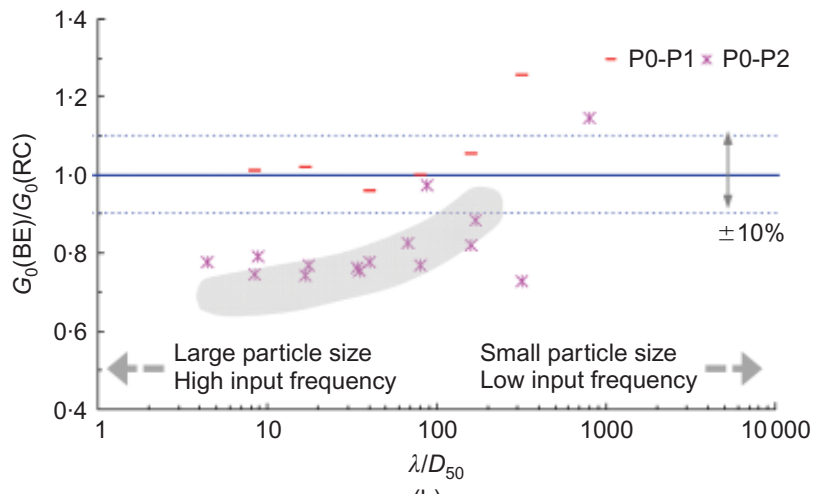

(b)

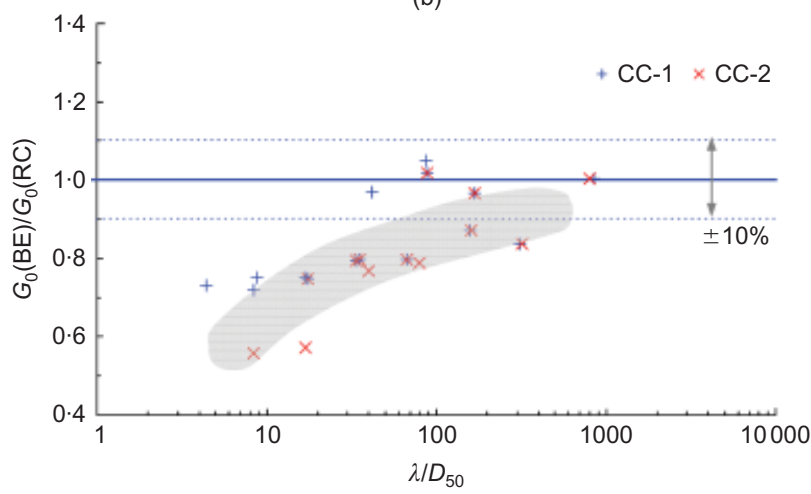

(c)

Fig. 16. Shear stiffness values estimated using various interpretation methods as a function of ratio between wavelength and particle size $\left(\sigma^{\prime}=100 \mathrm{kPa} ; e \approx 0 \cdot 584\right)$ : (a) start-to-start method; (b) peak-to-peak method; (c) cross-correlation method 
exhibit a tendency to decrease with $\lambda / D_{50}$ ratio, but the variation is approximately less than $10 \%$.

Based on a systematic examination of the characteristics of waveforms, and taking the $\mathrm{RC}$ measurements as a reference, the following strategies are recommended for conducting and interpreting $\mathrm{BE}$ tests for stiffness measurement.

(a) A range of excitation frequencies covering the threshold frequency should be used, and the waveforms at various frequencies should be examined as a whole.

(b) The excitation frequencies should be selected such that the ratio of travel distance and wavelength is between 2 and 4 (higher $R_{\mathrm{d}}^{\text {in }}$ values do not seem to help reduce the uncertainty).

(c) The start-to-start method using point $\mathrm{S} 2$ is recommended; for coarse materials where point S2 is not clear, point S4 is a reasonable alternative.

\section{COMPARISONS OF BE AND RC MEASUREMENTS}

A large number of $\mathrm{BE}$ and $\mathrm{RC}$ tests have been performed over a range of confining stresses and void ratios, and thereby offer an excellent opportunity to compare the stiffness values from the two methods. In doing so, the $\mathrm{BE}$ and $\mathrm{RC}$ measurements of the small-strain stiffness are shown against confining stress in Figs 17(a), 17(b) and 17(c) for specimens GB-A, GB-B and GB-D, respectively. It is evident that $G_{0}$ values estimated from both the $\mathrm{RC}$ and $\mathrm{BE}$ tests show an increase with the confining stress. As indicated by the trend lines for the upper and lower bounds, the stress dependence can be well represented by a power law, with the exponent being about $0 \cdot 4$. The data also indicate that at a given confining stress the $G_{0}$ value decreases with increasing void ratio. Using the empirical formula given in equation (1) to account for the effects of confining stress and void ratio, the two parameters $A$ and $n$ have been determined for the RC and BE measurements, respectively (see Table 3). For the purpose of comparison, values of the two parameters determined using test data for Toyoura sand (Yang \& Gu, 2010) are also included.

It is of interest to note from Fig. 17 and Table 3 that for fine granular materials (GB-D and Toyoura sand) the BE measurements are apparently greater than the $\mathrm{RC}$ measurements, whereas for coarse granular materials (GB-A) the two methods give $G_{0}$ values that are consistent overall. The results for the medium-coarse glass beads (GB-B) appear to be in between, with the $\mathrm{BE}$ measurements being slightly greater than the RC measurements. This marked feature can be found in an alternative way, as shown by Fig. 18, where the $\mathrm{BE}$ measurements of $G_{0}$ are plotted against the RC measurements. For both the fine and coarse specimens, the difference between the $\mathrm{BE}$ and $\mathrm{RC}$ measurements is approximately within $10 \%$, meaning that the effect of testing method is practically negligible.

Possible reasons for the finding that $\mathrm{BE}$ measurements of stiffness are larger than the corresponding ones from RC tests for fine and medium-coarse granular may be that: (a) the overall strain level involved in $\mathrm{BE}$ tests is somehow lower than that involved in RC tests; and (b) the RC test measures the overall stiffness of the specimen, whereas the $\mathrm{BE}$ test measures the central part of the specimen between the transmitter and receiver (Fig. 19), which tends to be stiffer than the whole specimen in the case of fine and medium-coarse granulates. Further work to explore the reasons behind this interesting observation would be of interest.

Figure 20 shows variations of stiffness values, determined by the $\mathrm{BE}$ and $\mathrm{RC}$ tests, with particle size at the dense, medium-dense and loose states. An alternative view of the

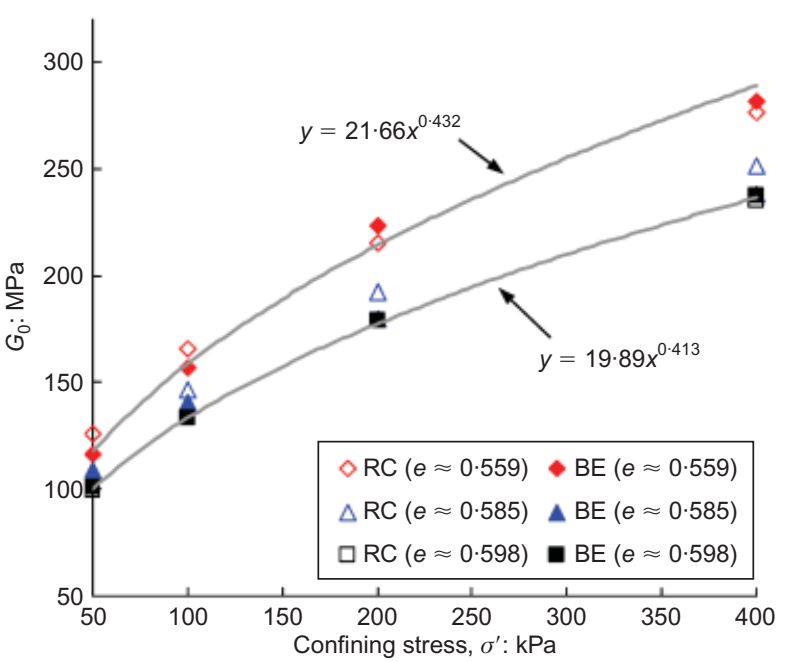

(a)

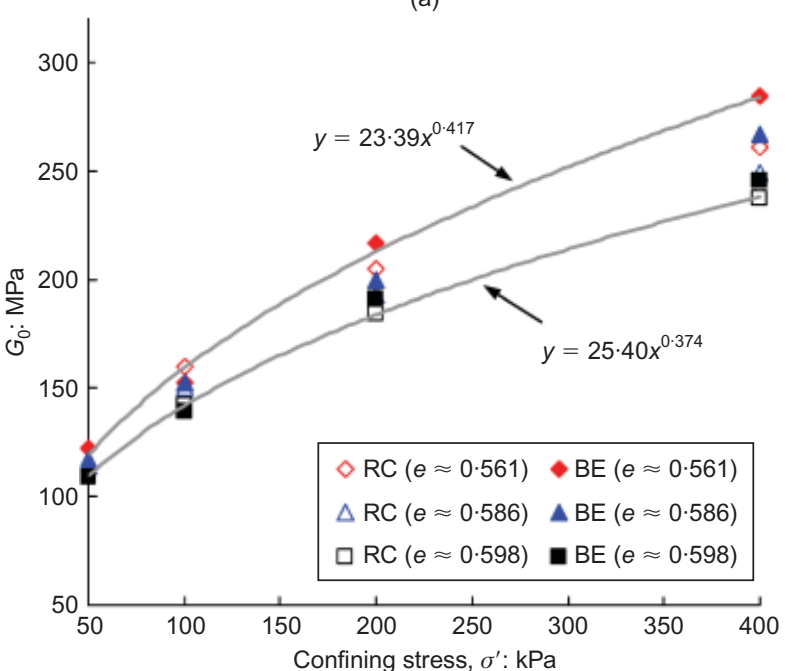

(b)

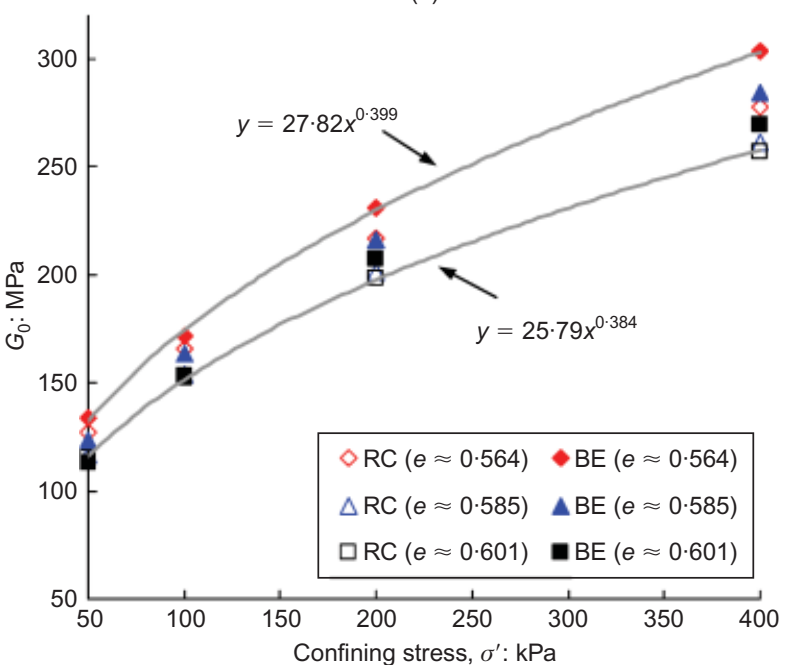

(c)

Fig. 17. Shear stiffness as function of confining stress: (a) GB-A, $D_{50}=1.750 \mathrm{~mm}$; (b) GB-B, $D_{50}=0.920 \mathrm{~mm}$; (c) GB-D, $D_{50}=$ $0 \cdot 195 \mathrm{~mm}$

results is given in Fig. 21, where the $\mathrm{BE}$ measurements normalised by the corresponding RC measurements are shown as a function of particle size. Both $\mathrm{BE}$ and $\mathrm{RC}$ measurements show a trend that $G_{0}$ values decrease slightly with mean particle size $D_{50}$, particularly for specimens at the loose state. However, given that the variations are approximately within $10 \%$, and given the uncertainty in- 
Table 3. Best-fit parameters for shear stiffness measurements

\begin{tabular}{|c|c|c|c|c|}
\hline \multirow[t]{2}{*}{ Material } & \multirow[t]{2}{*}{ Test method } & \multicolumn{3}{|c|}{ Parameters } \\
\hline & & $F(e)$ & $A$ & $n$ \\
\hline \multirow[t]{2}{*}{ GB-A } & $G_{0}(\mathrm{BE})$ & $(2 \cdot 17-e)^{2}$ & 88.91 & $0 \cdot 39$ \\
\hline & $G_{0}(\mathrm{RC})$ & $F(e)=\frac{(2 \cdot 1)-e)}{1}$ & $91 \cdot 22$ & $0 \cdot 40$ \\
\hline \multirow{2}{*}{ GB-B } & $G_{0}(\mathrm{BE})$ & $1+e$ & $90 \cdot 73$ & $0 \cdot 40$ \\
\hline & $G_{0}(\mathrm{RC})$ & & $87 \cdot 80$ & $0 \cdot 40$ \\
\hline \multirow[t]{2}{*}{ GB-D } & $G_{0}(\mathrm{BE})$ & & $97 \cdot 12$ & 0.41 \\
\hline & $G_{0}(\mathrm{RC})$ & & 92.79 & $0 \cdot 39$ \\
\hline \multirow[t]{2}{*}{ Toyoura sand } & $G_{0}(\mathrm{BE})$ & & $95 \cdot 10$ & $0 \cdot 41$ \\
\hline & $G_{0}(\mathrm{RC})$ & & $92 \cdot 50$ & $0 \cdot 41$ \\
\hline
\end{tabular}

Note: $A$ and $n$ are the two parameters in equation (1).

volved in the experiments and data interpretations, this modest size effect may, to a first approximation, be neglected: that is, the shear stiffness is assumed to be size independent for the range of particle sizes examined.

\section{MICROMECHANICAL CONSIDERATIONS}

From the micromechanical point of view, the small-strain shear stiffness of an assembly of particles should be closely related to the properties of discrete particles that interact with each other. To make this point, a grain-scale analysis is presented here. Note that this analysis is not aimed to explain the diverse features of the waveforms observed in the BE tests.

Consider a simple cubic array of identical spherical particles with dimension $l$, and subjected to an isotropic stress $\sigma^{\prime}$, as shown in Fig. 22. The number of spheres in each dimension is $m$, and the sphere is characterised by its radius $R$, Young's modulus $E_{\mathrm{g}}$, and Poisson's ratio $v_{\mathrm{g}}$ or shear modulus $G_{\mathrm{g}}\left(=E_{\mathrm{g}} / 2\left(1+v_{\mathrm{g}}\right)\right)$. Assuming that the Hertz-Mindlin contact law (Mindlin \& Deresiewicz, 1953; Duffy \& Mindlin, 1957) applies, the normal stiffness between two spheres that are in contact can be given as

$$
\begin{aligned}
& k_{\mathrm{n}}^{\mathrm{t}}=\frac{\mathrm{d} N}{\mathrm{~d} \varpi}=\frac{3}{4}\left(\frac{R^{1 / 2}}{\zeta}\right)^{2 / 3} N^{1 / 3} \\
& \zeta=\frac{3\left(1-v_{\mathrm{g}}^{2}\right)}{4 E_{\mathrm{g}}}
\end{aligned}
$$

where $N$ is the normal contact force between the two spheres, and $\bar{\omega}$ is the normal displacement. Similarly, the shear contact stiffness of the two spheres can be given by

$$
k_{\mathrm{s}}^{\mathrm{t}}=\frac{\mathrm{d} T}{\mathrm{~d} \delta}=\left(\frac{4 G_{\mathrm{g}}}{2-v_{\mathrm{g}}}\right)(\zeta R N)^{1 / 3}\left(1-\frac{T}{\mu N}\right)^{1 / 3}
$$

where $T$ is the shear contact force, $\delta$ is the tangential displacement between the spheres under shear, and $\mu$ is the friction coefficient between the two spheres. Note that for the cubic array the normal contact force $N$ can be calculated by

$$
N=4 \sigma^{\prime} R^{2}
$$

The normal contact stiffness and the tangential contact stiffness can be further expressed as

$$
\begin{aligned}
& k_{\mathrm{n}}^{\mathrm{t}}=A_{1}\left(\sigma^{\prime}\right)^{1 / 3} R \\
& k_{\mathrm{s}}^{\mathrm{t}}=A_{2}\left(\sigma^{\prime}\right)^{1 / 3} R\left(1-\frac{T}{\mu N}\right)^{1 / 3}
\end{aligned}
$$

where

$$
\begin{aligned}
& A_{1}=3\left[\frac{3\left(1-v_{\mathrm{g}}^{2}\right)}{E_{\mathrm{g}}}\right]^{-2 / 3} \\
& A_{2}=\frac{2}{2-v_{\mathrm{g}}}\left[12 G_{\mathrm{g}}^{2}\left(1-v_{\mathrm{g}}\right)\right]^{1 / 3}
\end{aligned}
$$

To determine the shear stiffness of the cubic array, a small shear stress increment $\Delta \tau$ is applied to the array, and the corresponding shear strain is then calculated from

$$
\begin{aligned}
\Delta \gamma & =\frac{4 m}{l} \frac{\Delta \tau R^{2}}{k_{\mathrm{s}}^{\mathrm{t}}} \\
& =\frac{4 m}{l} \frac{\Delta \tau R^{2}}{A_{2} R\left(\sigma^{\prime}\right)^{1 / 3}[1-(T / \mu N)]^{1 / 3}}
\end{aligned}
$$

The shear stiffness of the simple cubic array can then be given by

$$
G_{0}=\frac{\Delta \tau}{\Delta \gamma}=\frac{1}{2} A_{2}\left(\sigma^{\prime}\right)^{1 / 3}\left(1-\frac{T}{\mu N}\right)^{1 / 3}
$$

It is evident from equation (13) that the shear stiffness of the array is independent of the radius of the sphere. In other words, the particle size has no effect on the stiffness of the array.

\section{SUMMARY AND CONCLUSIONS}

This paper has identified and addressed two fundamental questions on the small-strain shear stiffness of granular material: particle size dependence and the effect of testing method. For three uniform types of glass beads with mean grain sizes varying from $0.195 \mathrm{~mm}$ to $1.750 \mathrm{~mm}$, series of $\mathrm{BE}$ and $\mathrm{RC}$ tests have been performed for a range of confining stresses and void ratios. Micromechanical modelling has also been conducted to offer an understanding from the grain scale. The key findings from the laboratory experiments are summarised as follows.

(a) The BE measurements of small-strain stiffness are comparable to the $\mathrm{RC}$ measurements, with differences being less than about $10 \%$. The BE measurements for the fine glass beads tend to be consistently larger than the resonant column measurements, especially at high confining stress levels, whereas this feature becomes less evident for the medium-coarse glass beads, and eventually diminishes for the coarse glass beads.

(b) The BE and RC tests both show a trend that the smallstrain stiffness $\left(G_{0}\right)$ decreases slightly with mean particle 


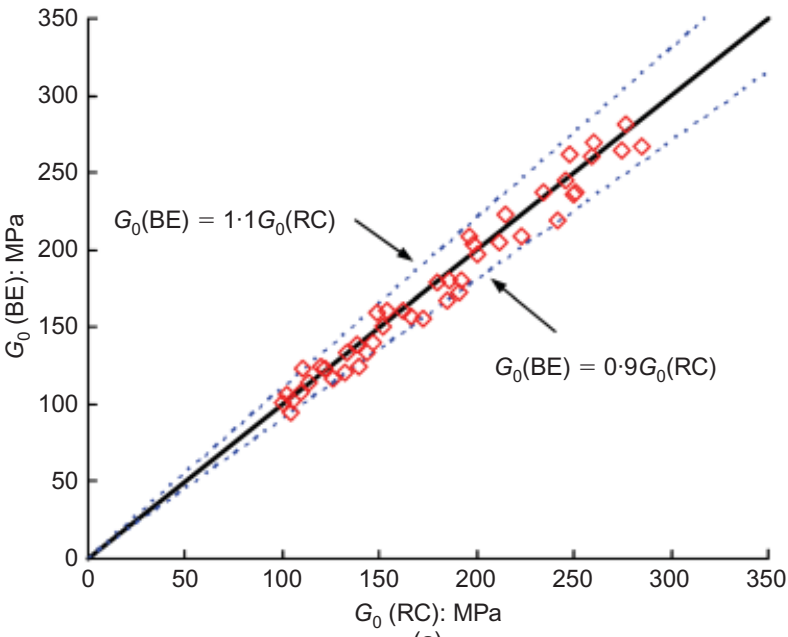

(a)

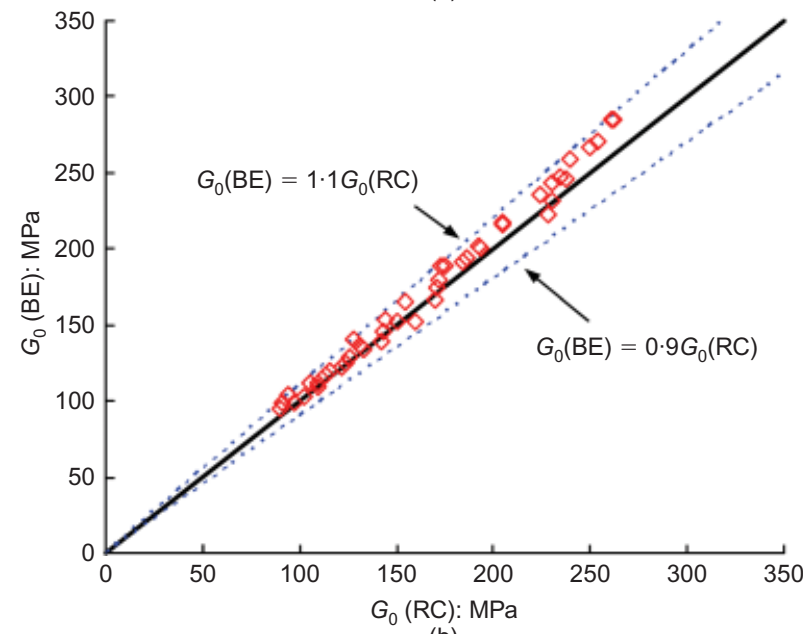

(b)

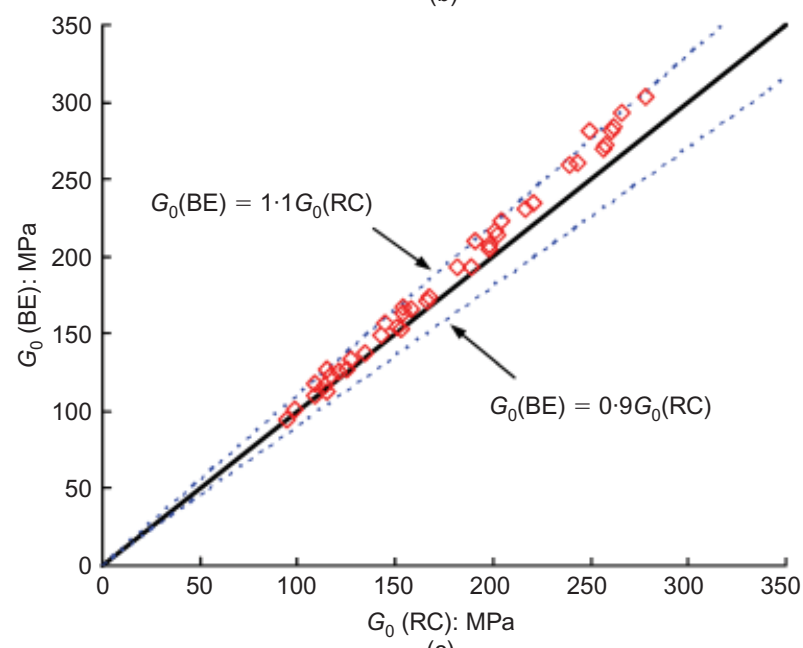

(c)

Fig. 18. Comparisons of shear stiffness measurements from BE and RC tests: (a) GB-A, $D_{50}=1.750 \mathrm{~mm}$; (b) GB-B, $D_{50}=0.920 \mathrm{~mm}$; (c) GB-D, $D_{50}=0.195 \mathrm{~mm}$

size $\left(D_{50}\right)$, particularly for glass beads at the loose state. Given that the variations in the measured stiffness values are generally small, and given the uncertainty in the laboratory experiments, it may be practically assumed that small-strain stiffness is size independent. This result is confirmed by the micromechanics-based analysis using the Hertz-Mindlin contact law.

(c) The waveforms generated in a granular specimen in $\mathrm{BE}$

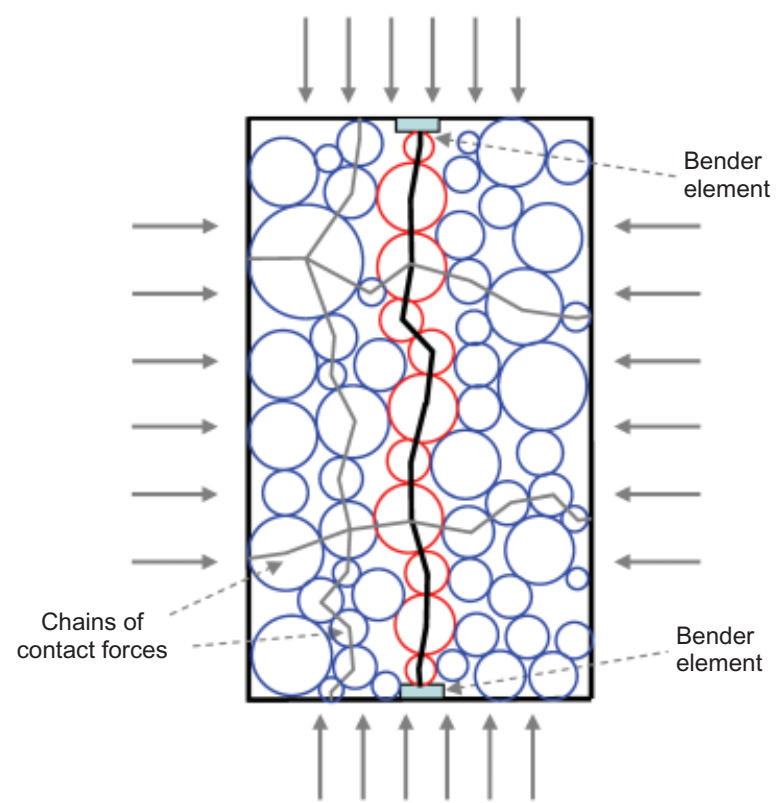

Fig. 19. Schematic illustration of wave paths and force chains in bender element tests

tests are complex, depending largely on the excitation frequency, the size of particles, and the confining stress level. The predominant frequency of the output signal tends to increase with excitation frequency, and then approach a limiting value at high frequencies. A threshold frequency marking this transition seems to exist, and both the threshold frequency and the limiting frequency show a tendency to increase with decreasing particle size.

(d) Under otherwise similar conditions, a decrease in particle size can result in high-frequency components in the output signal. The waveforms generated in fine granular specimens display a small-amplitude peak preceding the largest peak, and this feature tends to be enhanced at high frequencies and at large confining stresses. It is the excursion of the small peak rather than the largest peak that represents the true arrival of the shear wave.

(e) The conventional start-to-start method (using either point S5 or S4) tends to yield low stiffness values that decrease as the $\lambda / D_{50}$ ratio increases, meaning that the stiffness decreases with decreasing particle size for a given excitation frequency. The conventional peak-to-peak method (using point P2) and the cross-correlation method also give low stiffness values that, however, tend to increase as the $\lambda / D_{50}$ ratio increases, meaning that for a given excitation frequency the stiffness increases with decreasing particle size.

( $f$ ) The degree of scatter in stiffness measurements due to different interpretation methods for BE tests appears to depend on the travel distance-to-wavelength ratio $\left(R_{\mathrm{d}}^{\mathrm{in}}\right)$. For $R_{\mathrm{d}}^{\text {in }}$ values ranging from 2 to 4 , the scatter seems to be the smallest; higher $R_{\mathrm{d}}^{\text {in }}$ values do not seem to help reduce uncertainty or improve accuracy. In this optimal range of $R_{\mathrm{d}}^{\text {in }}$ values the start-to-start method using point $\mathrm{S} 2$ works well for fine granular material, whereas the start-to-start method using point S4 works well for coarse specimens in which point S2 appears to merge with point S4.

\section{ACKNOWLEDGEMENTS}

The work presented in this paper was supported by the University of Hong Kong under the Seed Funding for Basic 

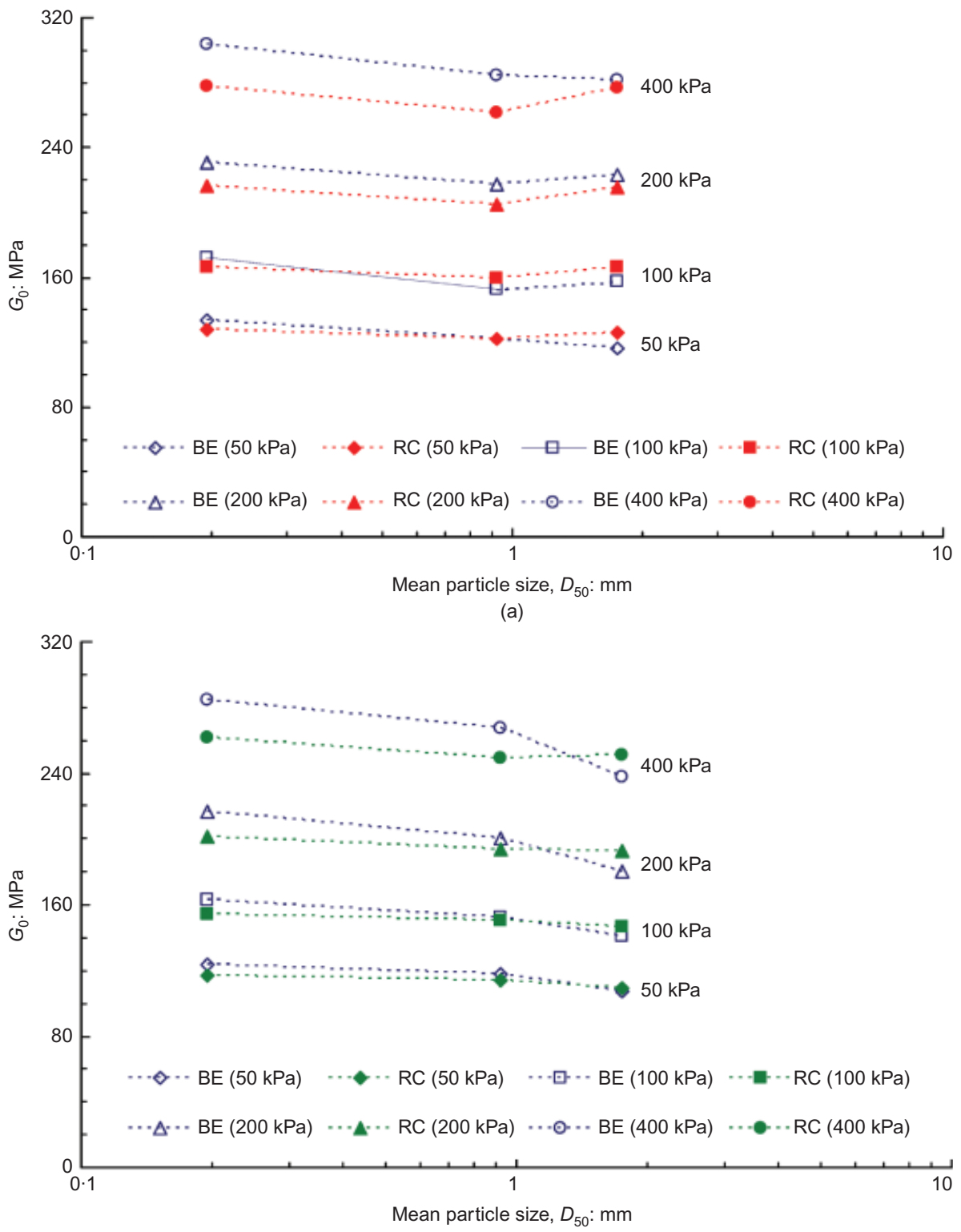

(b)

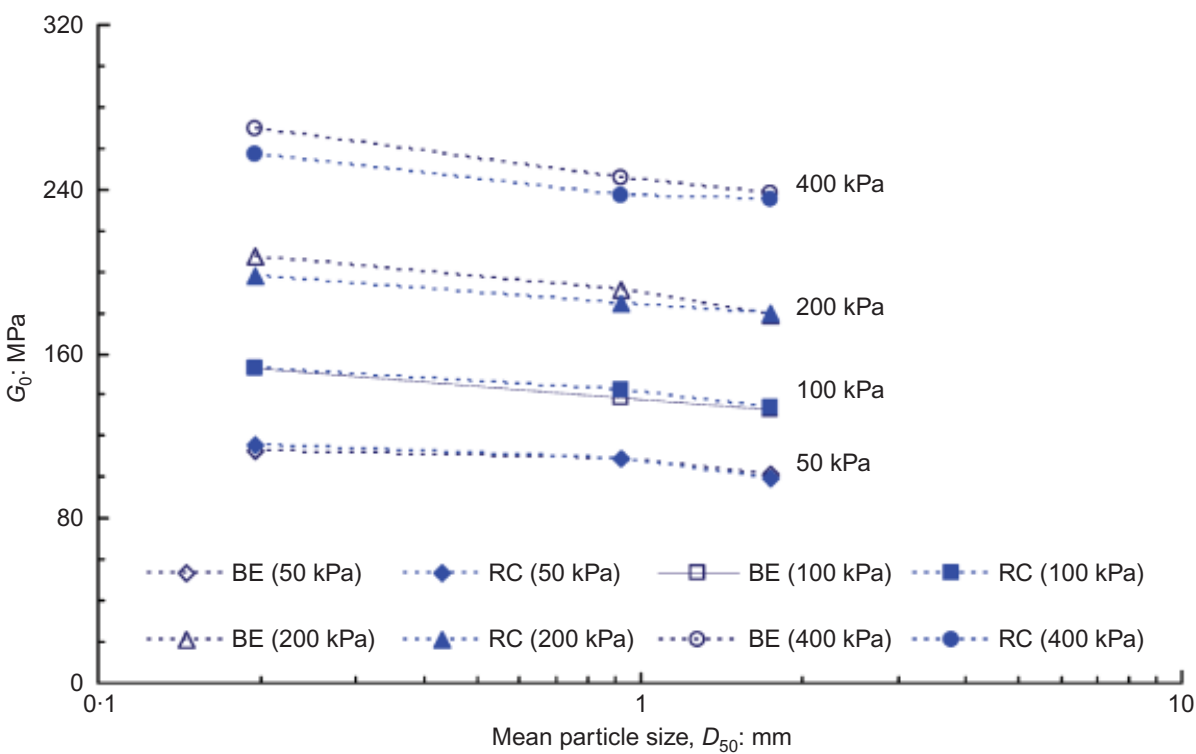

(c)

Fig. 20. Variation of shear stiffness with particle size: (a) dense state; (b) medium-dense state; (c) loose state 


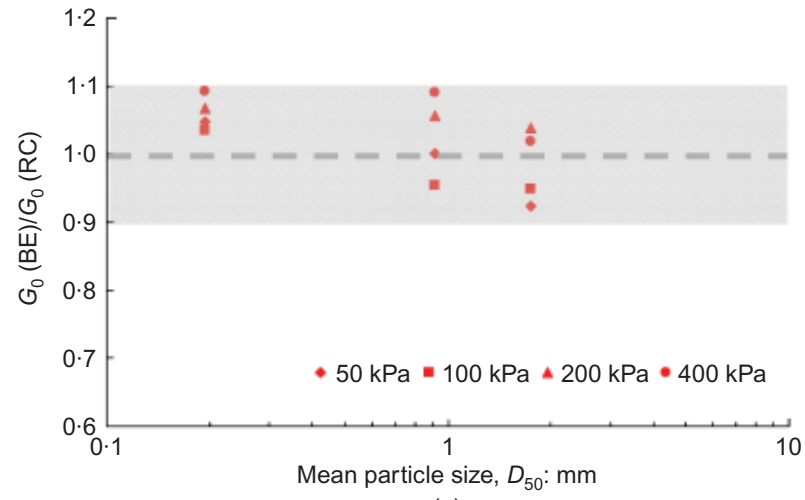

(a)

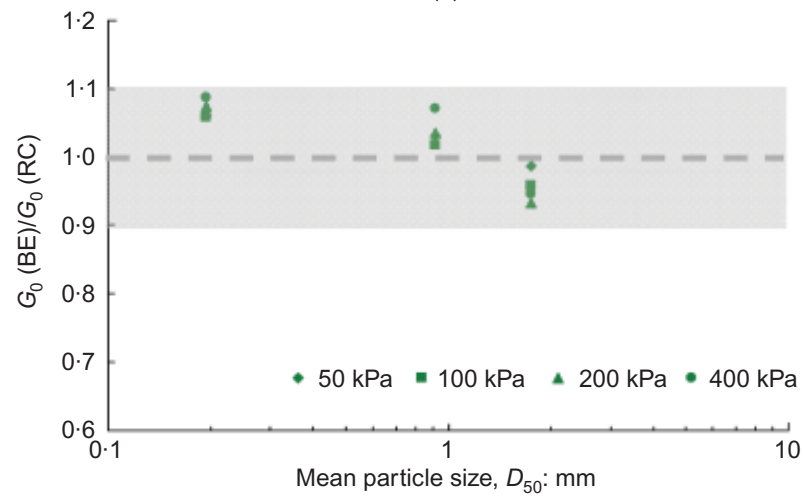

(b)

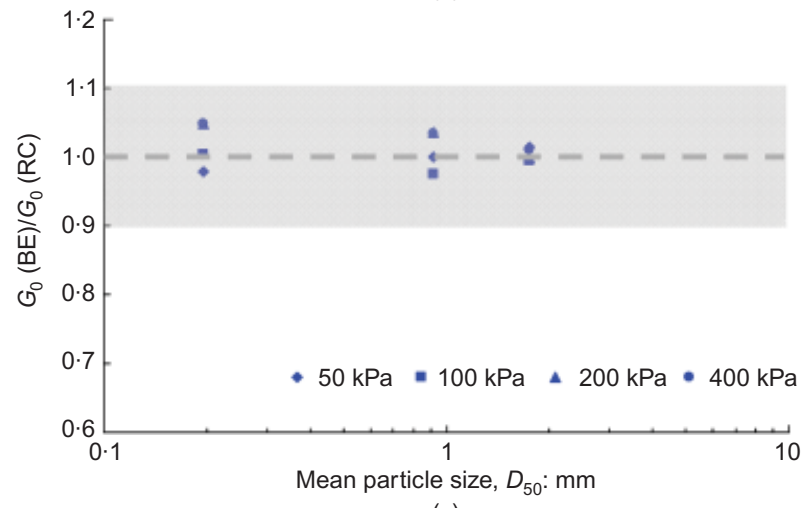

(c)

Fig. 21. Variation of shear stiffness ratio with particle size: (a) dense state; (b) medium-dense state; (c) loose state
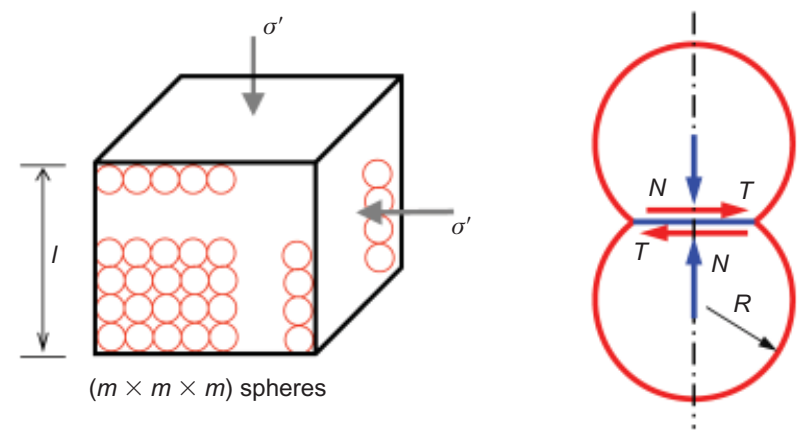

Fig. 22. A cubic array of spheres: Hertz-Mindlin contact model

Research scheme (11159098) and the Outstanding Young Researcher Award scheme (2006-2007). This support is gratefully acknowledged.

\section{NOTATION}

$A$ parameter in equation (1)

$A_{1}, A_{2}$ constants in equations (8) and (9)
$C_{\mathrm{u}} \quad$ coefficient of uniformity

$D_{10}$ particle size that $10 \%$ weight of soil are smaller than

$D_{50}$ mean particle size

$D_{60}$ particle size that $60 \%$ weight of soil are smaller than

$E_{\mathrm{g}} \quad$ Young's modulus of grains

$e$ void ratio

$F(e)$ void ratio function

$f_{\text {in }}$ frequency of input signal in BE test

$f_{\text {out }}$ predominant frequency of output signal in BE test

$G_{\mathrm{g}}$ shear modulus of grains

$G_{0}$ (or $G_{\max }$ ) soil small-strain shear modulus

$k_{n}^{\mathrm{t}}$ normal contact stiffness

$k_{\mathrm{s}}^{\mathrm{t}} \quad$ shear contact stiffness

$l$ length of simple cubic array

$L_{\mathrm{tt}}$ travel distance of shear wave

$m$ number of spheres in each dimension in simple cubic array

$N$ normal contact force

$n$ stress exponent

$p_{\mathrm{a}}$ reference stress

$R$ radius of sphere

$R_{\mathrm{d}}^{\text {in }}$ ratio of wave travel distance to wavelength based on input frequency

$T$ shear contact force

$V_{\mathrm{s}}$ shear wave velocity

$\Delta \gamma \quad$ shear strain induced by shear stress increment

$\delta$ tangential displacement between spheres under shear

$\lambda$ wavelength

$\mu$ friction coefficient between spheres

$\nu_{\mathrm{g}}$ Poisson's ratio of grains

$\rho$ mass density

$\sigma^{\prime} \quad$ effective confining stress

$\Delta \tau$ shear stress increment

$\varpi$ normal displacement between spheres under compression

\section{REFERENCES}

Bartake, P. P. \& Singh, D. N. (2007). Studies on the determination of shear wave velocity in sands. Geomech. Geoengng 2, No. 1, 41-49.

Brignoli, E. G. M., Gotti, M. \& Stokoe, K. H. II. (1996). Measurement of shear waves in laboratory specimens by means of piezoelectric transducers. Geotech. Test. J. 19, No. 4, 384-397.

Cho, G., Dodds, J. \& Santamarina, J. C. (2006). Particle shape effects on packing density, stiffness and strength: natural and crushed sands. J. Geotech. Geoenviron. Engng ASCE 132, No. 5, 591-602.

Chung, R. M., Yokel, F. Y. \& Drnevich, V. P. (1984). Evaluation of dynamic properties of sands by resonant column testing. Geotech. Test. J. 7, No. 2, 60-69.

Clayton, C. R. I. (2011). Stiffness at small strain: research and practice. Géotechnique 61, No. 1, 5-38, http://dx.doi.org/10. 1680/geot.2011.61.1.5.

Clayton, C. R. I., Priest, J. A., Bui, M., Zervos, A. \& Kim, S. G. (2009). The Stokoe resonant column apparatus: effects of stiffness, mass and specimen fixity. Géotechnique 59, No. 5, 429437, http://dx.doi.org/10.1680/geot.2007.00096.

Duffy, J. \& Mindlin, R. D. (1957). Stress-strain relations and vibrations of a granular medium. J. Appl. Mech. 24, 585-593.

Dyvik, R. \& Madshus, C. (1985). Lab measurements of $G_{\max }$ using bender element. Proceedings of the ASCE convention on advances in the art of testing soils under cyclic conditions, Detroit, MI, pp. 186-196.

Hardin, B. O. \& Richart, F. E. (1963). Elastic wave velocities in granular soils. J. Soil Mech. Found. Engng Div. ASCE 89, No. SM1, 39-56.

Iwasaki, T. \& Tatsuoka, F. (1977). Effect of grain size and grading on dynamic shear moduli of sand. Soils Found. 38, No. 1, $19-35$.

Jovicic, V., Coop, M. R. \& Simic, M. (1996). Objective criteria for determination of $G_{\max }$ from bender element tests. Géotechnique 46, No. 2, 357-362, http://dx.doi.org/10.1680/geot.1996.46.2. 357.

Lee, J. S. \& Santamarina, J. C. (2005). Bender elements: perform- 
ance and signal interpretation. J. Geotech. Geoenviron. Engng ASCE 131, No. 9, 1063-1070.

Lings, M. L. \& Greening, P. D. (2001). A novel bender/extender element for soil testing. Géotechnique 51, No. 8, 713-717, http://dx.doi.org/10.1680/geot.2001.51.8.713.

Lo Presti, D. C. F., Jamiolkowski, M., Pallara, O. \& Pedroni, A. C. S. (1997). Shear modulus and damping of soils. Géotechnique 47, No. 3, 603-617, http://dx.doi.org/10.1680/geot.1997.47.3.603.

Mindlin, R. D. \& Deresiewicz, H. (1953). Elastic spheres in contact under oblique forces. J. Appl. Mech. ASME 20, 327-344.

Patel, A., Bartake, P. P. \& Singh, D. N. (2008). An empirical relationship for determining shear wave velocity in granular materials accounting for grain morphology. Geotech. Test. J. 32, No. 1, 1-10.

Sanchez-Salinero, I., Roesset, J. M. \& Stokoe, K. H. II. (1986). Analytical studies of body wave propagation and attenuation, Report No. GR-86-15. Austin, TX, USA: University of Texas.

Sharifipour, M., Dano, C. \& Hicher, P. Y. (2004). Wave velocities in assemblies of glass beads using bender-extender elements.
Proc. 17th ASCE Conf. Engng Mech. (EM2004), University of Delaware.

Viggiani, G. \& Atkinson, J. H. (1995). Interpretation of bender element tests. Géotechnique 45, No. 1, 149-154, http:// dx.doi.org/10.1680/geot.1995.45.1.149.

Wichtmann, T. \& Triantafyllidis, Th. (2009). Influence of the grainsize distribution curve of quartz sand on the small strain shear modulus $G_{\max }$. J. Geotech. Geoenviron. Engng ASCE 135, No. $10,1404-1418$.

Yamashita, S., Kawaguchi, T., Nakata, Y., Mikami, T., Fujiwara, T. \& Shibuya, S. (2009). Interpretation of international parallel test on the measurement of $G_{\max }$ using bender elements. Soils Found. 49, No. 4, 631-650.

Yang, J. (2002). Liquefaction resistance of sand in relation to Pwave velocity. Géotechnique 52, No. 4, 295-298, http:// dx.doi.org/10.1680/geot.2002.52.4.295.

Yang, J. \& Gu, X. Q. (2010). Dynamic shear modulus of dry sand: effect of test method. Proc. 14th Eur. Conf. on Earthquake Engng, Ohrid, paper 368 (CD-ROM). 